\title{
Commercial U.S. Nuclear Reactors and Waste: The Current Status
}
A. M. Platt
J. V. Robinson

September 1980

Prepared for the U.S. Department of Energy under Contract DE-AC06-76RLO 1830

Pacific Northwest Laboratory Operated for the U.S. Department of Energy by Battelle Memorial Institute 


\title{
NOTICE
}

This report was prepared as an account of work sponsored by the United States Government. Neither the United States nor the Department of Energy, nor any of their employees, nor any of their contractors, subcontractors, or their employees, makes any warranty, express or implied. or assumes any legal liability or responsibility for the accuracy, completeness or usefulness of anv information, apparatus, product or process disclosed, or represents that its use would not infringe privately owned rights.

The views, opinions and conclusions contained in this report are those of the contractor and do not necessarily represent those of the United States Government or the United States Department of Energy.

PACIFIC NORTHWEST LABORATORY

operated by

BATTELLE

for the

UNITED STATES DEPARTMENT OF ENERGY

Under Contract DE-AC06-76RLO 1830

\author{
Printed in the United States of America \\ Available from \\ National Technical Information Service \\ United States Department of Commerce \\ 5285 Port Royal Road \\ Springfield. Virginia 22151
}

Price: Printed Copys

$\because$ Microfiche $\$ 3,00$

VTIS

-Pages Selling Price

$\begin{array}{lr}001-025 & \$ 4.00 \\ 026-050 & \$ 4.50 \\ 051-075 & \$ 5.25 \\ 076-100 & \$ 6.00 \\ 101-125 & \$ 6.50 \\ 126-150 & \$ 7.25 \\ 151-175 & \$ 8.00 \\ 176-200 & \$ 9.00 \\ 201-225 & \$ 9.25 \\ 226-250 & \$ 9.50 \\ 251-275 & \$ 10.75 \\ 276-300 & \$ 11.00\end{array}$




\title{
33679000544710
}

COMMERCIAL U.S. NUCLEAR REACTORS

AND WASTE: THE CURRENT STATUS

\author{
A. M. Platt \\ J. V. Robinson
}

September 1980

Prepared for

the U.S. Department of Energy

under Contract DE-ACO6-76RLO 1830

Pacific Northwest Laboratory

Richland, Washington 99352 


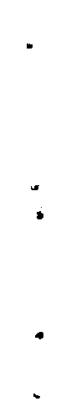




\section{CONTENTS}

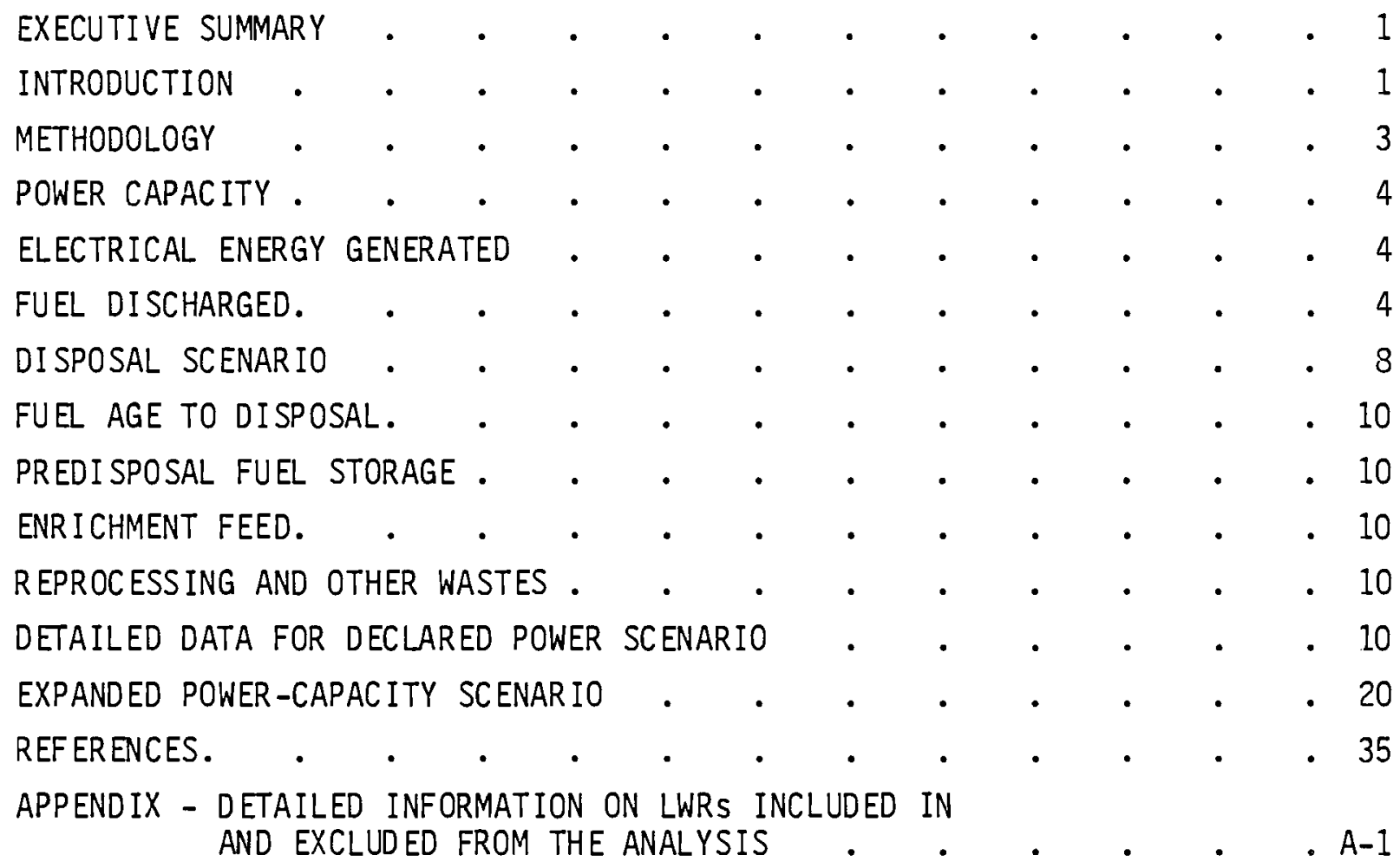




\section{FIGURES}

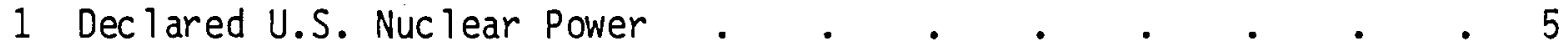

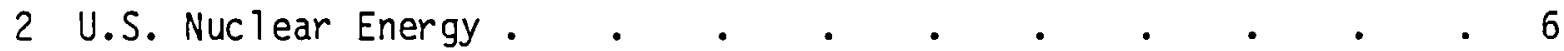

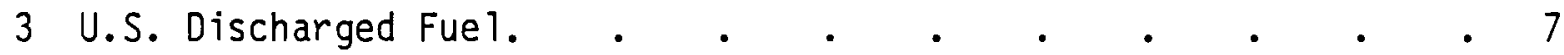

4 U.S. Fuel Disposed .

5 Minimum Age of Fuel Disposed .

6 Fuel in Predisposal Storage . . . . . . . . . . . . . 12

7 U.S. Nuclear Power - Expanded Scenario . . . . . . . . 21

8 U.S. Nuclear Energy - Expanded Scenario . . . . . . . . 23

9 U.S. Discharged Fue 1 - Expanded Scenario. . . . . . . . 24

10 U.S. Fuel Disposed - Expanded Scenario • • • • . . . 25

11 Minimum Age of Fue 1 Disposed - Expanded Scenario . . . . . 26

12 Fuel in Predisposal Storage - Expanded Scenario . . . . 27

\section{TABLES}

1 Disposal Scenario--Declared Power Capacity . $\quad$. $\quad$. $\quad$. $\quad$ - 8

2 Projected Volumes of Transuranic Waste Disposed in Geologic Repository from the LWR-Rcycle Fuel Cyc le.

3 Projected Low-Level Waste Volumes from the

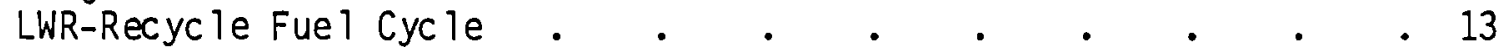

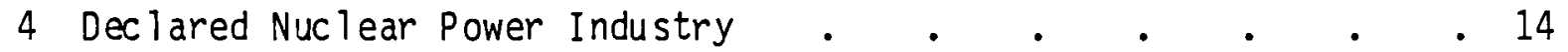

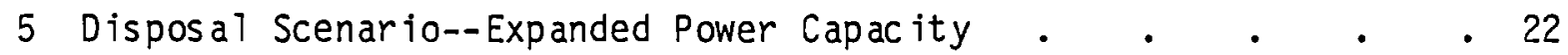

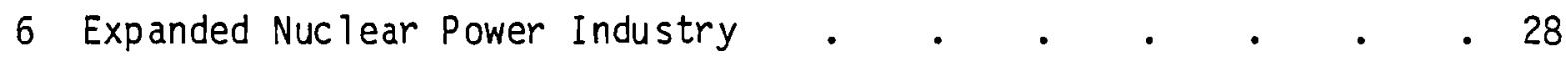

7 Comparison of the Two Power Scenarios . . . . . . . . 34

A-1 U.S. LWR Commercial Reactors - PWR (On-Line/Down) . . . . A-1

A-2 U.S. LWR Commercial Reactors - PWR (Projected) . . . . A-2

A-3 U.S. LWR Commercial Reactors - PWR (Cancelled) . . . . A-4

A-4 U.S. LWR Commercial Reactors - PWR (Indefinite) . . . . A-5

A-5 U.S. LWR Commercial Reactors - BWR (On-Line/Down) . . . . A-6

A-6 U.S. LWR Cormercial Reactors - BWR (Projected) . . . . A-7

A-7 U.S. LWR Conmercial Reactors - BWR (Cancelled) . . . . A-8

A-8 U.S. LWR Commercial Reactors - BWR (Indefinite) . . . . A-9

A-9 U.S. LWR Commercial Reactors (Announced as of 1-1-77, Since Cancelled) 


\section{EXECUTIVE SUMMARY}

Between March 1 and June 15, 1980, the declared(a) size of the commercial light waste reactor (LWR) nuclear power industry in the U.S. has decreased another 9 GWe.

For the presently declared size:

- The 165 declared reactors will peak at a capacity of 153 GWe in 2001 and will consume about 870,000 MTU as enrichment feed.

- The theoretical rate of enrichment requirements will peak at about $19,000,000 \mathrm{SWUs} / \mathrm{yr}$ in the year 2014 .

- As few as two repositories each with capacity equivalent to 100,000 MTU would hold the waste.

- Predisposal storage reactor basins and AFRs (away-from-reactor basins) would peak at $<85,000$ MTU in the year 2020 if the two repositories were cormissioned in the years 1997 and 2020 .

It should be noted that the number of declared LWRs has dropped from 226 on December 31, 1974 to 165 as of this writing. The oil equivalent of the energy loss, assuming a $50 \%$ efficiency in use as in cars, is 17,000 million barrels. This is about 10 years of the current rate of U.S. consumption of OPEC Oil.

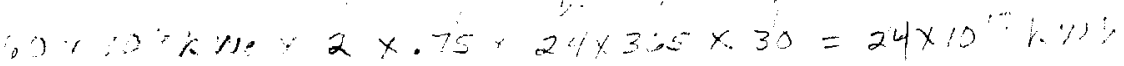

\section{INTRODUCTION $24,3, \cdots$,}

In April of this year, PNL published the first (PNL-3317-1) in this series of reports intended to track implications to DOE's commercial nuclear waste

(a) Power plants that either have operated, are operating, are being built, or have been announced. 
management program due to the recent rapidly decreasing size of that industry. The present report, the second in the series, differs from the first in four ways:

1. Five boiling water reactors (BWRs)--Hartsville A1, A2, B1, B2, and Phipps Bend 2--and two pressurized water reactors (PWRs)--Cherokee 3 and Yellow Creek 2--have been withdrawn from the list of reactors previously announced as planned to be built and have been placed in the indefinite category.

2. Two reactors (Millstone 1 and 2) are now planned to be permanently shut down earlier than anticipated, thus decreasing slightly the integrated nuclear electric energy that will be produced.

3. Thirty-three other reactors in the declared category are now projected to be on-line later than previously anticipated.

4. The report format has been changed so that the tabular data and the associated graphs for the two power capacity scenarios are now brought together in such a way as to make the report easier to use and the scenarios easier to compare.

This report provides an executive overview of the LWR industry in the U.S. and its implications to the Department of Energy's (DOE) commercial waste management program. It sumarizes the status of the LWR commercial reactors and spent fuel in the U.S. as of mid-CY-1980.

Two projections (scenarios) are made to the year 2060. One relates to the presently declared size of the industry and assumes that no new plants will be announced. The second relates to the situation if a resurgence in the industry were to occur, resulting in some additional plants being built. The analysis of this enhanced scenario illustrates the analytical capability used in producing this report.

This report is based on the status of the commercial nuclear power industry as of Apri1 1, $1980^{(1)}$ except that the power reactors cancelled between then and June 15, 1980, as reported in Nucleonics Week and other trade 
publications, have been deleted from the two scenarios analyzed. Detailed information on the PWRs and BWRs excluded from, as well as those included in, the analyses is provided in the Appendix.

It should also be noted that only commercial LWR reactors are included. Reactors specifically excluded are: Hallam, EBR-2, Hanford N, Enrico Fermi-1, Fort St. Vrain, Peach Bottom-1, Carolina-Virginia Tube Reactor, and Piqua.

For convenience, all logistics for the fuel cycles are expressed in metric tons of uranium or heavy metal (MTU/MTHM). (The information in Tables 2 and 3 $c$ an be used to convert MTHM to volumes of waste generated in the various fue 1 cycle operations.)

\section{METHODOLOGY}

The information contained in this report is a very simple extrapolation of the past performance of existing U.S. light water reactors. The computer program used assumes that the announced future reactors will be operated simi1 arly. More sophisticated computer codes ${ }^{(2,3)}$ attempt to anticipate changes in fuel management for existing reactors as well as the operation of announced reactors on almost a batch-by=batch basis.

The program used in producing the results in this report is based on the logic that reactor fue 1 management in BWRs and PWRs has progressed to the point where it is very closely tied to the electrical energy produced. Thus the reactor startup and shutdown times establish the quantity and timing of fue 1 discharge. Hypothetical reactors $c a n$ be added to the stream by defining their capacities and startup and shutdown times.

With fuel discharge cycles established, the timing and size of terminal storage needs are clearly projected for any given power capacity scenario. Utilizing the program, combinations of various power scenarios, fuel exposures, and repository construction schedules can be analyzed very rapidly. 


\section{POWER CAPACITY}

Figure 1 shows cumulative power capacity of reactors in the U.S. whose status ranges from public announcement to those that have been or are now in conmercial operation. This is the declared power scenario. Note that these reactors attain a peak generating capacity of 153 GWe at the turn of the century.

Reactor lifetimes are arbitrarily assumed to be 40 years unless contrary specific data are available.

\section{ELECTRICAL ENERGY GENERATED}

Figure 2 shows the cumulative electrical energy generated when 50, 60, or $70 \%$ of the installed capacity is used. The actual energy generated in the past (see squares in Figure 2) is also displayed. The actual energy curve closely matches the $60 \%$ capac ity parameter.

\section{FUEL DISCHARGED}

The quantities of fuel that will be discharged by the nuclear power industry will be influenced primarily by the fraction of installed capacity used and by the exposure of the fuel. These two factors are combined in the exposure/on-line parameters $(a)$ shown in Figure 3 . This figure projects the cumulative fuel to be discharged by the nuclear capacity previously described. Actual fuel discharged through 1979 is only slightly $(<7 \%)$ overestimated by the $15,000 \mathrm{MWED} / \mathrm{T}-\mathrm{OL}$ parameter. ${ }^{(4)}$ It is expected that future reactor operations

(a) The burnup parameter, MWED/T-OL, will be unfamiliar to most readers. It is the electrical energy that would be generated by a metric ton of nuclear fue 1 if $100 \%$ of the available power capacity were used. For example,

$$
31,500 \frac{\mathrm{MWD}}{\mathrm{Ton}} \text { (therma1) } \times \frac{1 \text { electrical }}{3 \text { therma }} \times \frac{100 \% \text { available }}{70 \% \text { on line }}=15,000 \mathrm{MWED} / \mathrm{T}-0 \mathrm{~L}
$$

Dividing this parameter into the maximum possible energy production by installed nuclear capacity yields amount of fuel discharged. 


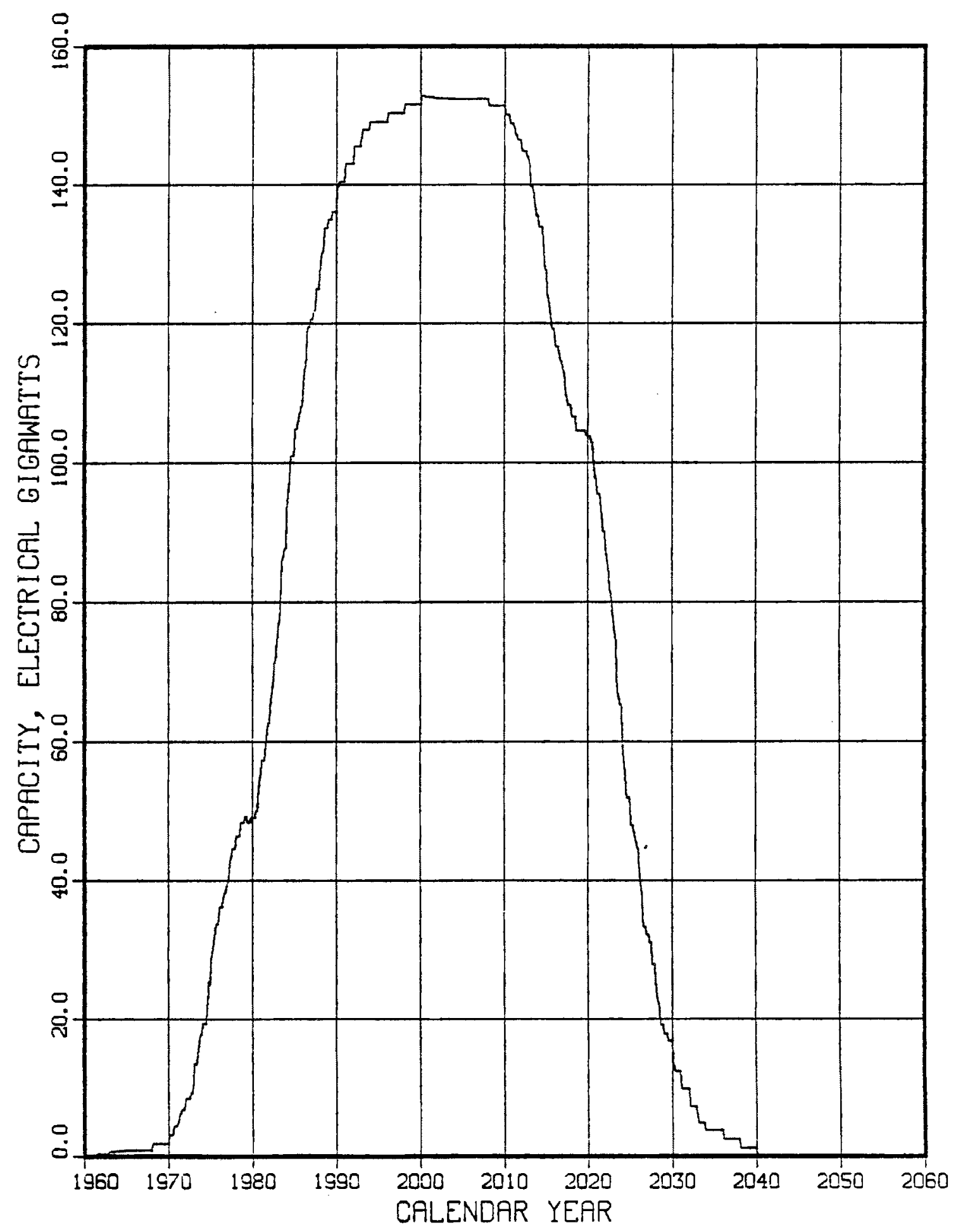

FIGURE 1. Declared U.S. Nuclear Power Reactor Status 06/15/80 


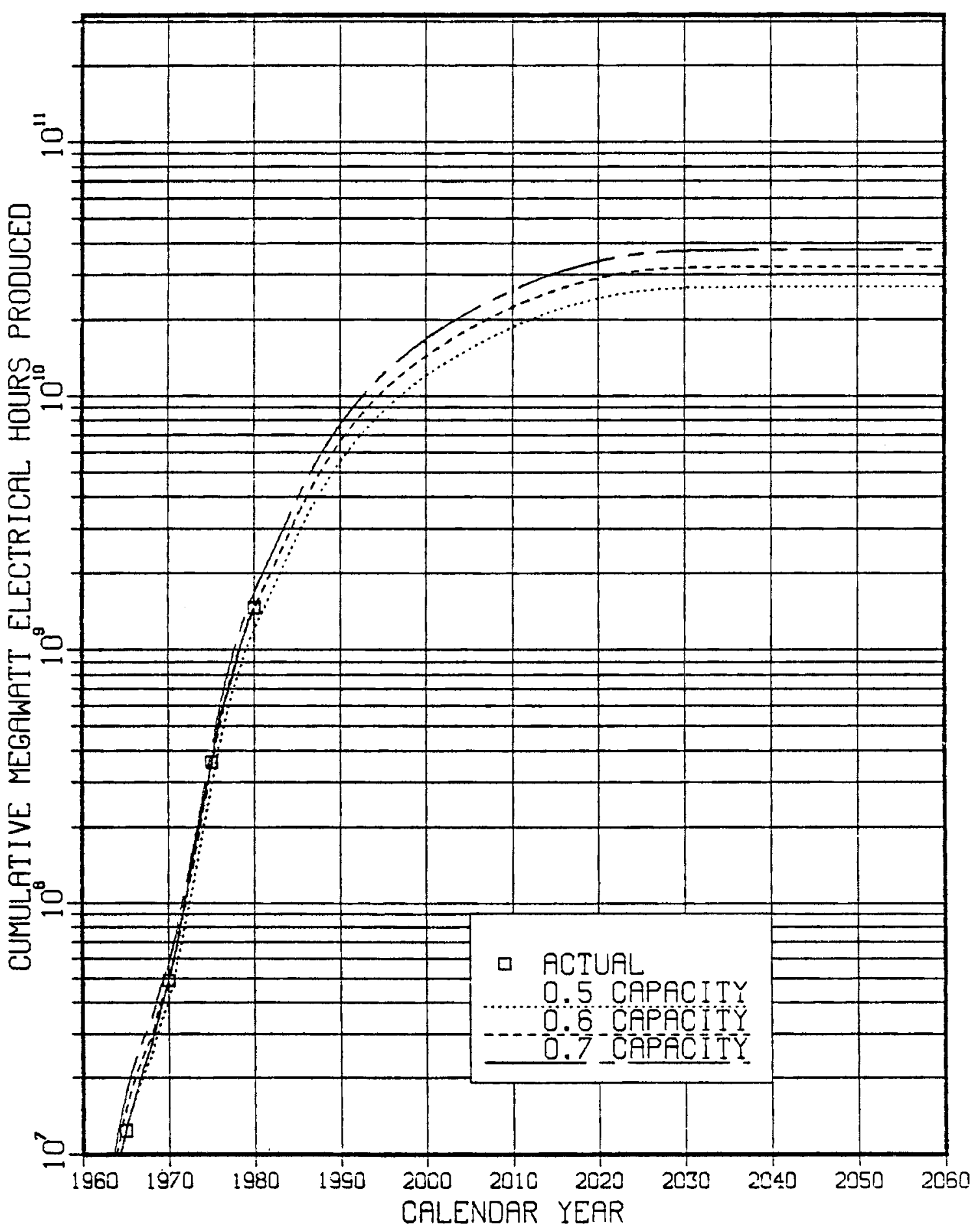

FIGURE 2. U.S. Nuclear Energy -

Reactor Status 06/15/80 


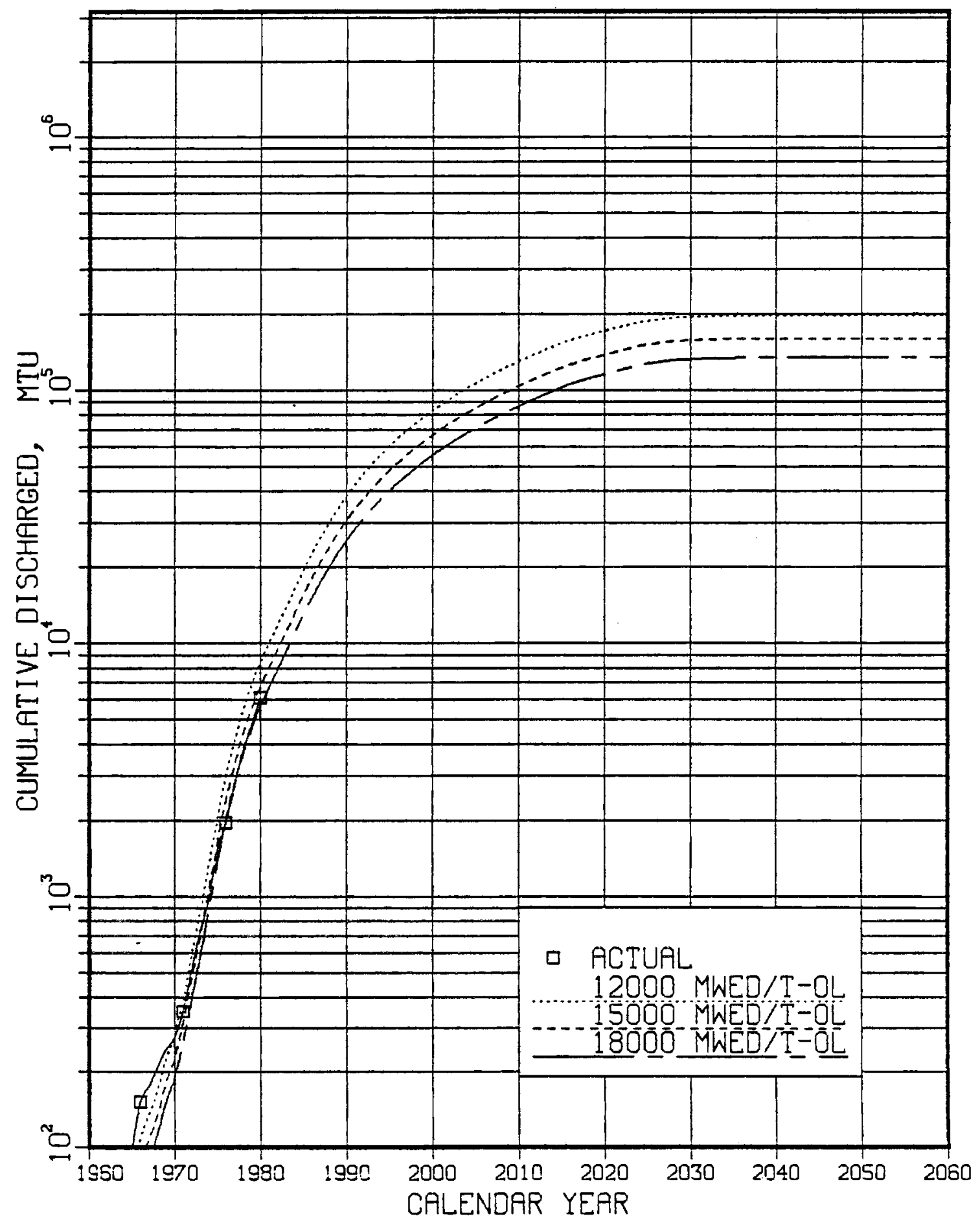

FIGURE 3. U.S. Discharged Fue1 -

Reactor Status 06/15/80 
wi1l be at higher exposures and capacity. Recent data indicate that fuel to be discharged in the future may better be estimated by the 18,000 MWED/T-OL parameter.

If waste packages were comprised of one element from the predominant PWRs or three elements from the BWRs, some 2000 packages would be generated from 1000 MTU.

\section{DISPOSAL SCENARIO}

No firm schedule has been established for the operation (loading rate, capacity) and startup of repositories for storage of waste from the commercial nuclear power industry. For this analysis, we assumed that the repositories would be built and operated according to the schedule shown in Table 1. You will note that the repositories begin operation in different years and each uses a lower loading rate in the first two years of operation than that used for the remaining years of its operation.

The cumulative amount of spent fuel disposed in these repositories is shown in Figure 4.

\section{TABLE 1. Disposal Scenario--Declared Power Capacity}

\begin{tabular}{|c|c|c|c|c|c|}
\hline $\begin{array}{c}\text { Repository } \\
\text { Identification }\end{array}$ & $\begin{array}{l}\text { Datel (a) } \\
\text { of } \\
\text { Startup } \\
\end{array}$ & $\begin{array}{c}\text { Date of } \\
\text { Shutdown } \\
\text { or Changed } \\
\text { Operation } \\
\end{array}$ & $\begin{array}{c}\text { Maximum } \\
\text { Loading Rate, } \\
\text { MTU/yr }\end{array}$ & $\begin{array}{c}\text { Spent Fuel } \\
\text { Loaded in } \\
\text { Time Period, } \\
\text { MTU } \\
\end{array}$ & $\begin{array}{c}\text { Capacity, } \\
\text { MTU } \\
\end{array}$ \\
\hline \multirow[t]{3}{*}{ Rep 1} & 1997.0 & 1998.0 & 1,000 & 1,000 & \multirow{3}{*}{98,000} \\
\hline & 1998.0 & 1999.0 & 2,000 & 2,000 & \\
\hline & 1999.0 & 2037.0 & 2,500 & 95,000 & \\
\hline \multirow[t]{3}{*}{ Rep 2} & 2020.0 & 2021.0 & 1,000 & 1,000 & \multirow{3}{*}{75,500} \\
\hline & 2021.0 & 2022.0 & 2,000 & 2,000 & \\
\hline & & 2051.0 & 2,500 & 72,500 & \\
\hline
\end{tabular}

(a) Throughout tables herein, years or dates are expressed in decimal fashion; thus 1980 or 1980.0 is January 1 of 1980 . 


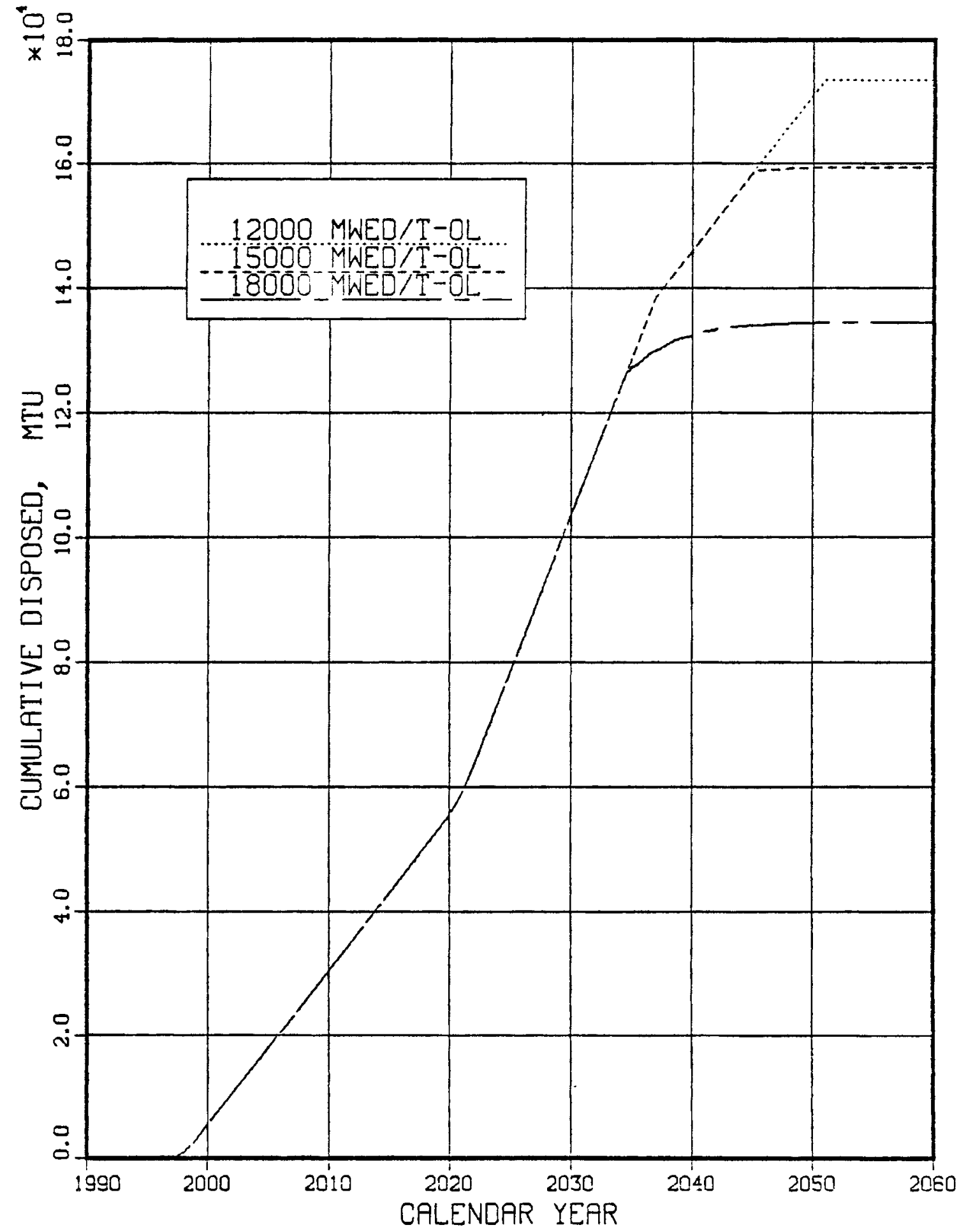

FIGURE 4. U.S. Fuel Disposed -

Reactor Status 06/15/80

Disposal Start 1997 


\section{FUEL AGE TO DISPOSAL}

Significant quantities of spent fuel first became available in the U.S. in the mid-1960s. This 20-year-old material could be used to start operation of the repositories. After that, and based on the principle of oldest fuel to disposal first, the age of the youngest fuel going to disposal was determined and is shown in Figure 5 for the repository disposal scenario assumed.

PREDISPOSAL FUEL STORAGE

Predisposal storage will peak at about 82,000 MTHM (for the 15,000 MWED/ T-OL parameter) in 2020. Quantities of fuel in predisposal storage at other times are shown in Figure 6. A one-time correction to fuel stored has been

made (in 1973) for the 255 tons of commercial LWR fuel reprocessed by Nuclear Fuel Services, Inc., at West Valley, NY, in the period 1967 to 1971 . No corrections have been made for the minute amounts of fuel consumed in R\&D operations, e.g., the Nuclear Waste Vitrification Project conducted at PNL.

\section{ENRICHMENT FEED}

As a sidelight to these calculations, it was determined that some 817,000 MT of uranium feed to enrichment would be required for this scenario, with $0.2 \%$ tails and $3 \%$ product.

\section{REPROCESSING AND OTHER WASTES}

Table 2 shows the volume of TRU waste that would go to geologic disposal if the option to reprocess and recycle were elected rather than the once-through fuel cycle. Also LLW volumes for the recycle option are shown in Table 3.

\section{DETAILED DATA FOR DECLARED POWER SCENARIO}

The data (tabular and by year) from which Figures 1-6 were produces are given in Table 4. 


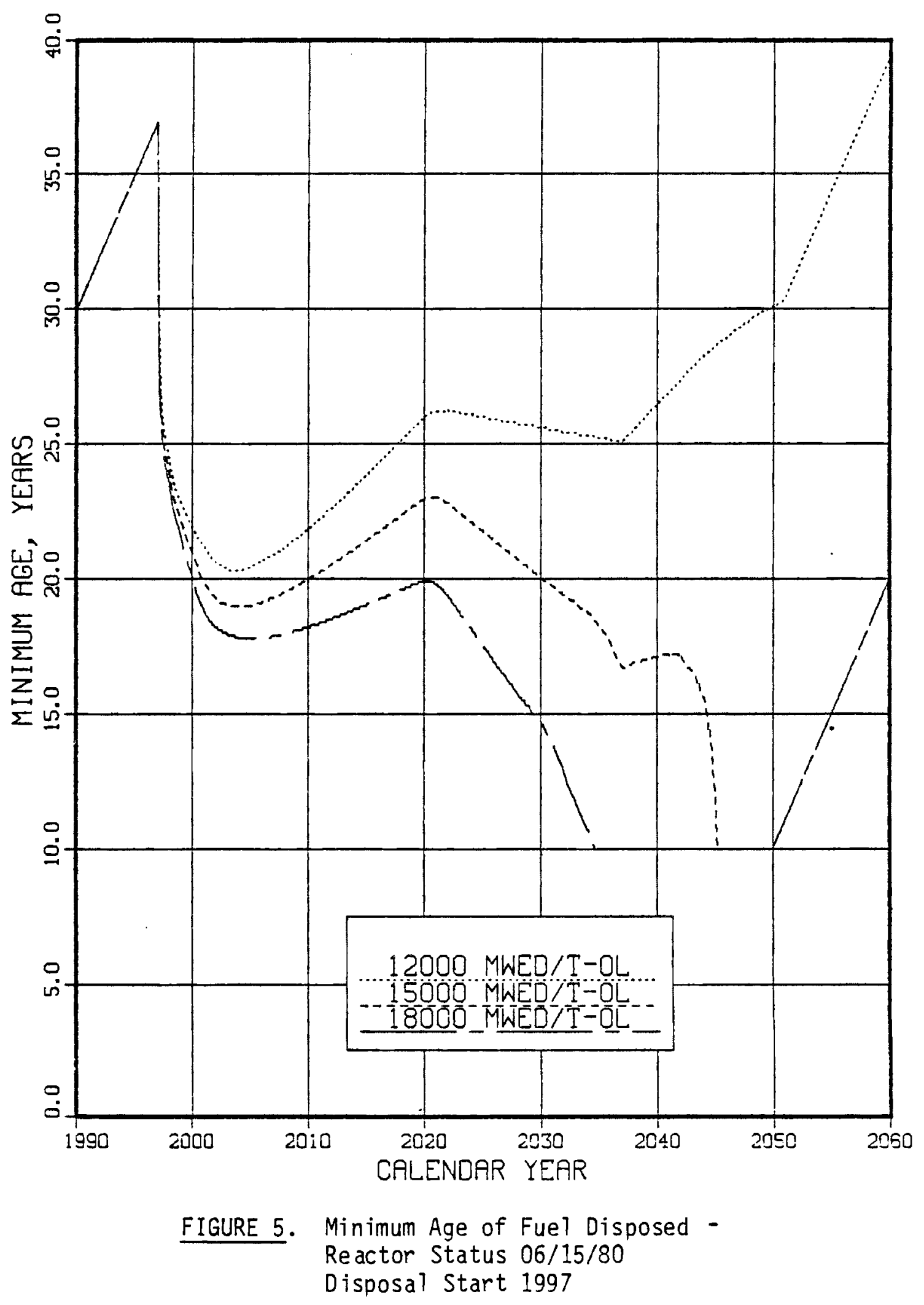




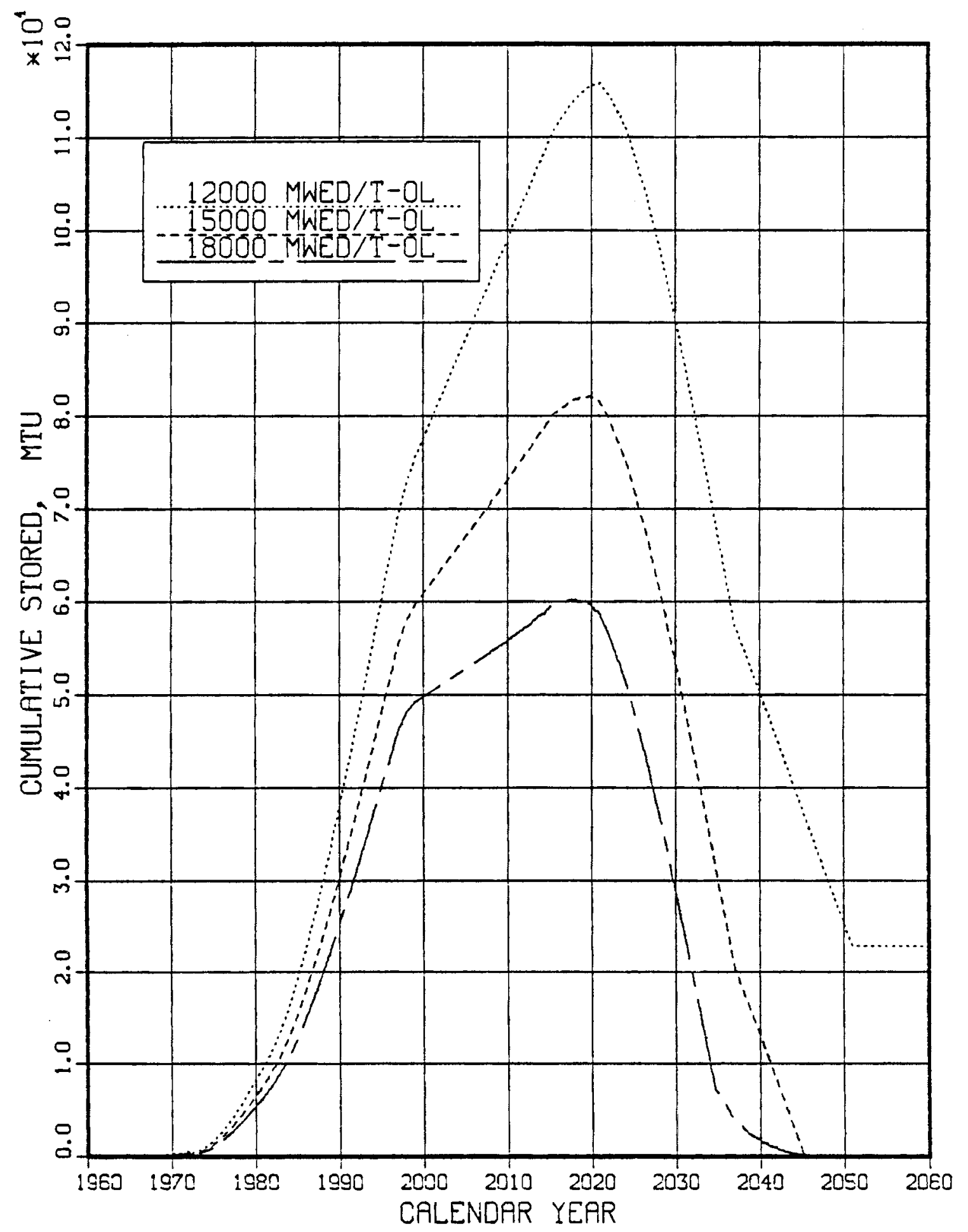

FIGURE 6. Fue 1 in Predisposal Storage Reactor Status 06/15/80 
TABLE 2. Projected Volumes of Transuranic Waste Disposed in Geologic Repository from the LWR-Recycle Fuel Cycle

Fue 1 reprocessing wastes

Vitrified HLW

Volume, $\mathrm{m}^{3} / 1000$ MTHM

Hulls and hardware 1600

Other TRU wastes

1200

Mixed-oxide fuel refabrication wastes 320

1900

Notes: Includes decommissioning wastes.

Fuel cycle character istics are those described in Ref. 5. Data for waste volumes were taken from Ref. 6 . Waste treatment assumptions include:

HLW: vitrification

General trash: incineration/cementation Wet wastes:

Scrap: cementation direct packaging

TABLE 3. Projected Low-Level waste Volumes from the LWR-Recycle Fuel Cycle

Mill tailings

Refinery, conversion and enrichment wastes

Uranium fuel fabrication wastes

Reactor wastes

Fuel reprocessing plant LLW

Tota 1
Volume, $\mathrm{m}^{3 / 1000 \mathrm{MTHM}}$ 950,000

680

900

32,000

630

984,000

Notes: Includes decomissioning wastes. Waste volume data were taken from Refs. 6 through 10. Uranium mill tailings volume assumes an ore containing 0.2 wt\% U with a $95 \%$ extraction efficiency. Waste treatment assumptions include:

General trash: compaction Wet wastes:

Scrap:

cementation direct packaging 
TABLE 4. Declared Nuclear Power Industry

REAT.TMR STATHS MG/15/a Acrual. II)

DATE

"ÂA"

1963.0
1961.0

1963.1
1963.0

1964.0

1965.0

1966.n

19k1.0

I 9 KA.

1969. n

1970.0

$1971 . n$

$1972 . n$

$1973 . n$

1974.0

1975.0

1976.0

1977.0

1979.0

19an.ก

|9A|. n

laA?.n

1983.n

1984.0

1985.0

$198 \% .0$

$1987 . n$
198.0.

1989.0

$1990 . n$

1991.0

1992.0

$1993 . n$

1994.0

$1995 . n$

$1996 . n$

1998.0

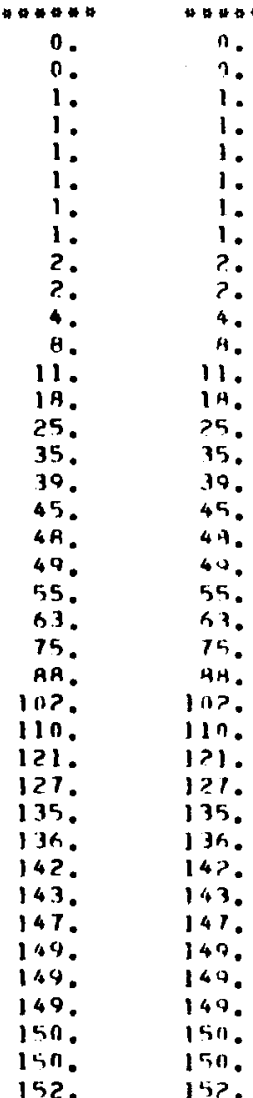

$$
\text { p } 0
$$$$
\text { if } F \text { a }
$$$$
\text { P }
$$

GO PER CFNT RO PEP CENT TOPER CENT ACTIIAL"

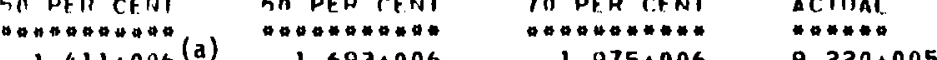

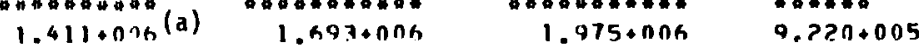

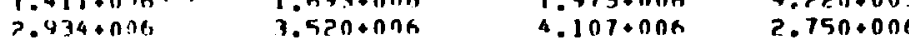

5.073+0NG G.0A7+016 $7.192+006 \quad 5.190+006$

R.5nT+nOG $1.021+0 n 7 \quad 1.191+007 \quad$ A.A4 1.006

$\begin{array}{llll}1.230+007 & 1.476+1007 & 1.722+007 & 1.240+007\end{array}$

$\begin{array}{llll}1.62 n+007 & 1.9510007 & 7.377+007 & 1.640+007\end{array}$

$\begin{array}{llll}2.075+007 & 2.430+007 & 3.936+007 & 2.160+007\end{array}$

$\begin{array}{llll}2.420+017 & 3.903+007 & 3.387+007 & 2.710+007\end{array}$

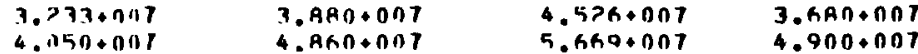

$4.1150+0117$

$5.6411+0 n 7 \quad 6.816+0 n 7$

A.475.nAT $1.017+n \cap B$

$1 ., 240+0,0)$

1.91400 ONA

1.4 A, 011 A

?. $303+008$

$3.445+108$

$5.123+10 n A$

7. กบP 0118

$9.37 n+00 A$

$1.186+009$

1.443 .009

$1.713+n n 9$

$? .17>6+109$

?. $394+1009$

2. $475 \cdot 0109$

$3.354+109$

$3.715+1799$

$4.532+100$

$5.141+109$

5. A76.10\%

h. 50n+ 109

1..3? ?.

A. nADC 0ก?

A. ALT + กO

$1.041+010$

1.n4lonis

$1.119+n 10$

$1.27 \mathrm{H}+11 \mathrm{~B}$

$7.457+107$

$1.1 A K+D O A$

$1.736+10 n 8$

$?$

$5,477+0 \cap A$

ค. $>74+$ TRA

$1.1193+0 \cap 9$

1. $783+n \cap 9$

$1.4 A 3+n 09$

$1.99 A+B \cap 9$

?. $364+10 n 9$

$? .794+109$

$3.307+1004$

4 . 5 A +009

$5.787+109$

ค. $045+100$

A. A55 000

7. AAA+109

a. 5 Sintong

A.

1.123 .010

$1.173+n 15$

$.675+194$

I. $7 n k+01 n$

$1.065+010$

$1.357+110$

1070010

$.491+n 10$
$1.544+010$

$.900+1007$
$.960+007$

$1.070+00 \mathrm{~A}$

$1.620+00 \mathrm{O}$

$2.440+008$

$3 . A \cap \cap+\cap O B$

$5.790+1008$

$3.910+0 \cap A$

$9.520+0 \pi)^{2}$

$1.210+109$
$1.470+109$

(a) Throughout these tables and elsewhere in the text, the convention for depicting very large numbers is $1.411+006=1.411 \times 10^{6}$.

MTII DISCHARGED AT MWEO/T-OL

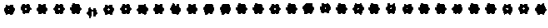

12000 15nOD JAONO ACTUAL.

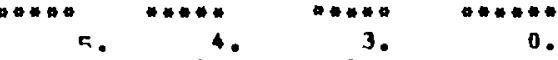

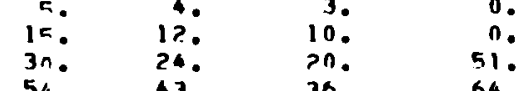

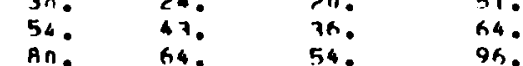

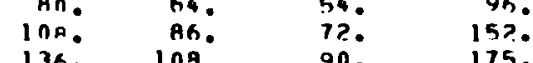

138. 109. 130.0175.

228: lAO. 151. 287.

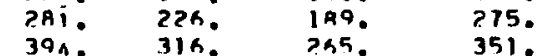

58A. 477. 194. 518.

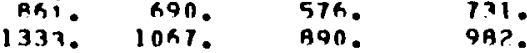

PnIF. 1617. 1352. 1534.

P9AK. 2394. 2010. 1962.

$413 n$. 3310. 2764. 2743.

5444. 4365. 3643. 3760.

GAAR. 5517. 46ח2. 4AR?

6553. 6141.

IIA17. 9469. 7996.

13947.11173 .9376$.

16497. 1321?. 11025.

19497. 19516. 130rA.

2P74L. lR214. IST

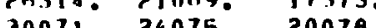

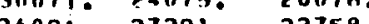

34091. 27291. ग255

4050 34014

$46849077496^{\circ} 31262$.

51285. 41047 14271

44654. 3727?

633? 4A2A4. 40252

64A7ח. 51914. 43777.

74AP? 59275. 46378

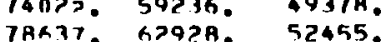


TABLE 4 (contd)

fi $4 \mathrm{~F}$ DATE ACTUAL TOTAL 2000.0 152.

2001.0

2001.0

2003.0

2004.0

2005.0

2006.0

2007.0

2008.0

2009

2010.0

2011.0

2011.0

2013.0

2014.0

$2015 . n$

2016.0

2017.0

2018.0

2019.0

ज
RFACTIR SIATUS NG/15/AA

115 N II L L A

$$
\text { M H H } A T
$$

$$
\text { P } \cap \text { W } P
$$

50 PER CENT

70 PER R.ENT

a* *.

$1.577+010$

$1.770+010$

$.064+010$
$1.95 A+010$

$2.051+010$

$2.145+010$

2. $33+010$

$3.733+010$

$2.425+110$

$2.51 A+010$

$3.611+010$

$? .703+010$

$? .793+010$

2. $A$ A? 010

$2.96 x+010$

$3.047+010$

$3.121+010$

$3.197+010$

$7.259+010$

$3.324+010$

3. TAR+ 010

$3.451+010$

$3.50 A+010$

$3.559+010$

$3.501+010$

$3.534+010$

$3.663+010$

3. KA5 010

$3.703+010$

3.7150110

$3.726+010$

$3.134+1110$

$3.74 n+010$

$3.744+010$

$3.747+110$

3.749 .010

$3.75 ?+010$

$3.757+010$

?. $6 A D+010$

$? .5 A 1+0111$

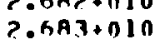

$3.755+010$
$3.756+010$

ACTUAL

MTII DISTHARGED AT MWEO/T-OI.

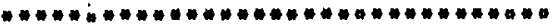

$12000 \quad 15000$ IANOO ACTUAL

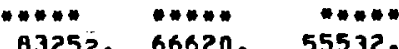

A791\% 70354 5AR46.

9257\%: 74082. 61754.

9721\%. 77796. B4B49.

101863 . A1513. 67948.

10650? A5226. 71042 .

11114? A893A, 74135.

115783.92650 . 77229.

$12049 \%$ 9 9430. 80390 .

125100.100118 .

12979A. In3A93. B6624.

13446月. 1 1 7653. B9776.

$13917 \% 111464.92991$.

14390R. 11531A. 96259.

$14848 \%$. 119080 . 99442.

153125. 122A97. 102746.

157324. 126360. 105717.

1610A9. 129422. 108310.

164A51. 132516. 110960 .

168190. 1352?1. 113236.

171427 . 137A15. 115406.

175n7n. 140839. 118019.

17A4lK. 143617. 12041A.

181734. 146434. 1?2A99.

184919. 149195. 125378.

18922\%. 151304. 12726\%.

19089R. 154346. 129977.

$192300.155578,131090$.

1933A7. 156528. 131956.

194?15. 157252. 1325n9.

194A2?. 1577A4. 133091.

195795. 15A195. 13346?.

195669 . 15A576. 133764.

195AAR. 15A7J6. 133935.

19R005. 15AA10. 136013.

196>07. 15A9AR. 134176.

19K?AK. 159051. 1342PA.

19644R. 15919A. 134365.
$1964 A 7.159230 .134391$. 
TABLE 4 (contd)

REACTMR STATUS OG/15/80

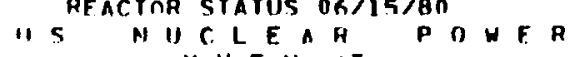

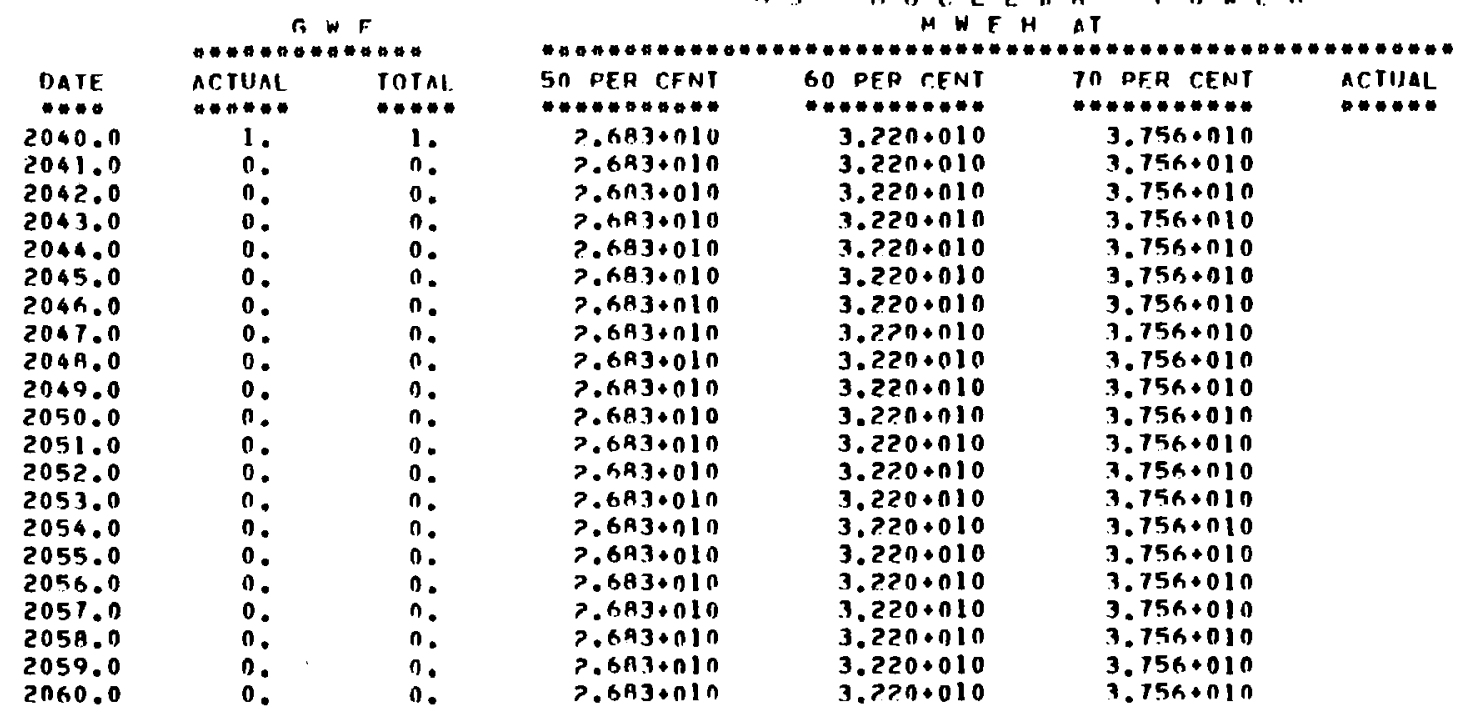

MTU DISCHARGET AT MHET/T-O

\begin{tabular}{|c|c|c|}
\hline 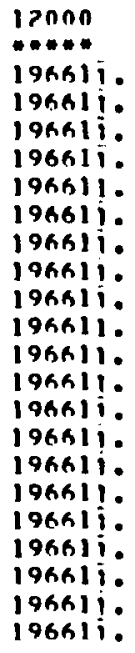 & $\begin{array}{l}15000 \\
6.15 \% \\
159346 . \\
159346 . \\
159346 . \\
159346 . \\
159346 . \\
159346 . \\
159346 . \\
159346 . \\
159346 . \\
159346 . \\
159346 . \\
159346 . \\
159346 . \\
159346 . \\
159346 . \\
159346 . \\
159346 . \\
159346 . \\
159346 . \\
159346 . \\
159346 .\end{array}$ & $\begin{array}{r}18000 \\
1345 n 3 . \\
134503 . \\
1345030 \\
134503 . \\
134503 . \\
1345 n 3 . \\
134503 . \\
134503 . \\
134503 . \\
134503 . \\
1345 n 3 . \\
134503 . \\
134503 . \\
134503 . \\
134503 . \\
134503 . \\
1345 n 3 . \\
134503 . \\
134503 . \\
1345 n 3 . \\
134503 . \\
1345 n 3 .\end{array}$ \\
\hline
\end{tabular}


TABLE 4 (contd)

RFACTOR STATUS OG/15/AO

FUFI STATIS, MTU

15.000 MWEN/T GL SCENARIN

RUMIII A TIVE

PREDIsposal

IERMINAL

CAPACIIY MINTMUM

STORAGE STORAGE

SINRAGE

MIUIYR CAPACTIY

AFE, YRS

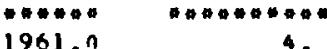

1961.0

$1963 . n$

1964.0

1965.0

1966.0

1967.0

1968.0

1969.0

1969.0

1971.0

1972.0

1973.0

1974.0

1975.0

1977.0

1978.0

979.0

1980.0

1981.0

1912.0

$1983 . n$

1984.0

1985.0

$19{ }^{2} \cdot{ }^{2}$

$1987 . n$

1988.0
1989.0

1989.0

1990.0

992.0

$1993 . n$

1994.0

1995.0

1996.0

1997.0

1998.0

1999.0

12

12

24.

64.

A6.

34.

134.
1 An.
226.

226.

316.

690.

1n67.

$1617^{\circ}$

2394 。

$331 n$.

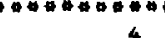

12.

43.

Bh.

InS.

134.

140.

316.

47 ?

435.

A) 3 .

1353.

2140 .

3056 .

4110.

776 ?

AnI7.

9469.

3212 .

15616 .

ค214.

$? 1069$

24075

27291.

30594 .

$34 \cap 14$.

37496.

41047.

44654

4 A2 4

51914.

55575 .

59236 .

6?.02H.

9714

$\ln 918$.

12958.

17961.

?חA15.

23月?0.

2.71136.

30340.

33759.

37>4?

4079 ?

$444 \pi 1 \%$

4 AO 30 .

$5160_{10} 0$.

55370.

57981.

59673.

$n$.
$n$.

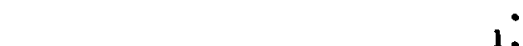

1.9

3.9

4.9

6.9

7.9
A. 9

9.9

10.9

12.9

13.9

14.9

15.9

16.9

17.9

1 $\mathrm{A.9}$

19.9

21.9
21.9

??.9

23.9

24.9

25.9
26.9

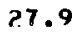

2ค. 9

79.9

$3 n .9$

31.9

32.9

33.9

34.9

$0 .:$
lonn:
30nn.

1000.

2000.
$250 n$.

Inno.

zono.
zann.

36.9

24.1
27.3 
TABLE 4 (contd)

HFACTOP STATUS 06/15/AO

FUE. STATIIS. MTU

15,000 MHED/T-OL SCENARIO

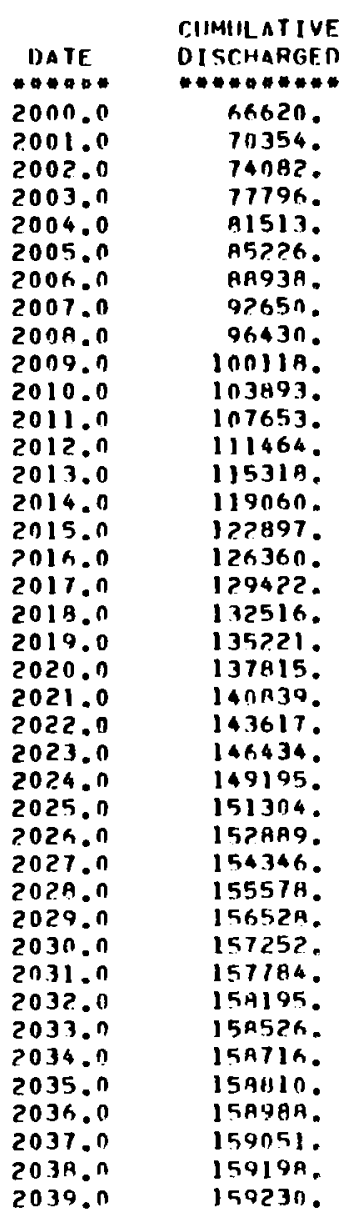

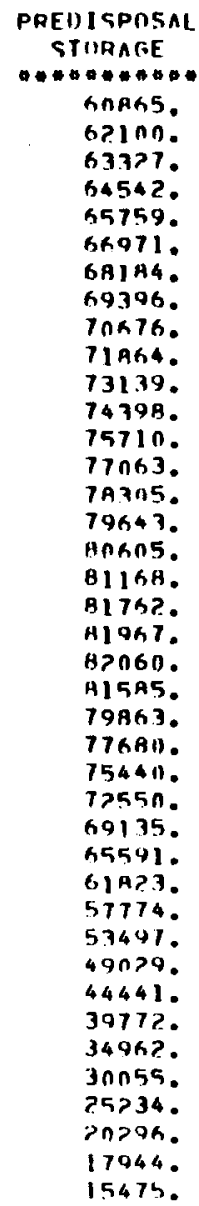

TERMINAL

SINRAGF.

......."

5500.
BnOn.

10500 .

13000 .

13500 .

18000.

ج950त.

23000.

28000.

30500 .

33000 .

35

.

4 .

43010.

45500

50500 .

.

53100.

55son.

53500

63500
68500

78500

73500

83500.

AP500.

93500.

98500.

103500 .

108500 .

$11350 n$.

114500

113500
123500.

123500.

133500 .

13 4500.

141000 .

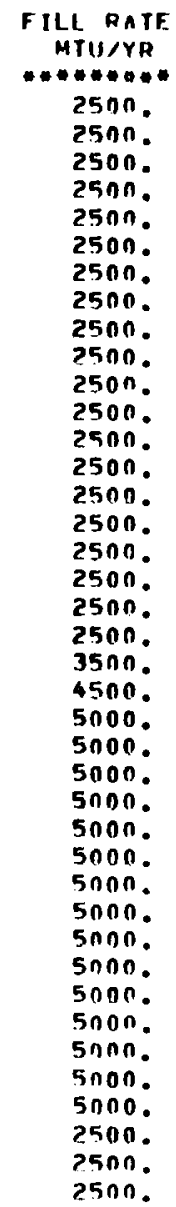

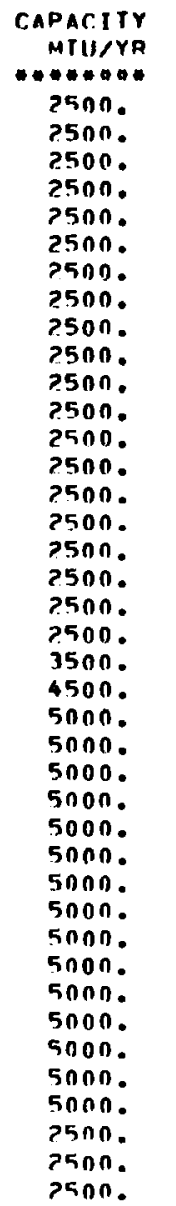

MINIMUM AGE, YAS 
TABLE 4 (contd)

PFACINR STATIIS NG/15/AN

FUFI STATIIS. MTII

15, OON MWEDTTOL SCENARIO

2
DAIF
2040.0
2041.0
2042.0
2043.0
2044.0
2045.0
2046.0
2047.0
2048.0
2049.0
2050.0
2051.0

CIIMIII A TIVE

DI EChapgen

PREIJISPOSAL

SIISPOSAL
SIIRRAGE

TERMINAL

159346 .

159346 .

antonat

STORAGE

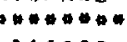

146000.

151000.

$1535 n n$.

159346.

In 592 .

A09?.

156000.

158500.

159346 .

3092.

$59 ?$.

159346 .

159346.

15B9AA.

(1)

FILL RATE

MINIMUIM

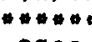

2500 .

mrii/Yr

159346.

59198 .

$1593 n^{\circ}$

159346.

2500 .

2500.

action

2500.15500 .16 .6

25nn. 15.6

159346 .

12.0

10.0

10.0

10.0

10.0

11.0 


\section{EXPANDED POWER-CAPACITY SCENARIO}

For comparison, it was assumed that an additional 120 GWe will be added to the declared power scenario. We assumed the additional power would be added between 1997 and 2010 at a rate approximating that expected to be added between the years 1980 and 1990. This power scenario is shown in Figure 7 .

For this expanded nuclear capacity, the loading schedule, shown in Table 5, for four repositories was assumed.

The energy produced, fuel discharged, fuel disposed, etc., for this power scenario are shown in Figures 7 to 12 and are on the same bases as for the declared power scenario previously described. The detailed data from which Figures 7 through 12 were produced are given in Table 6 .

The two scenarios can be compared as shown in Table 7 to the year 2060 using the 15,000 MWED/T-OL and 60\% capacity parameters. 


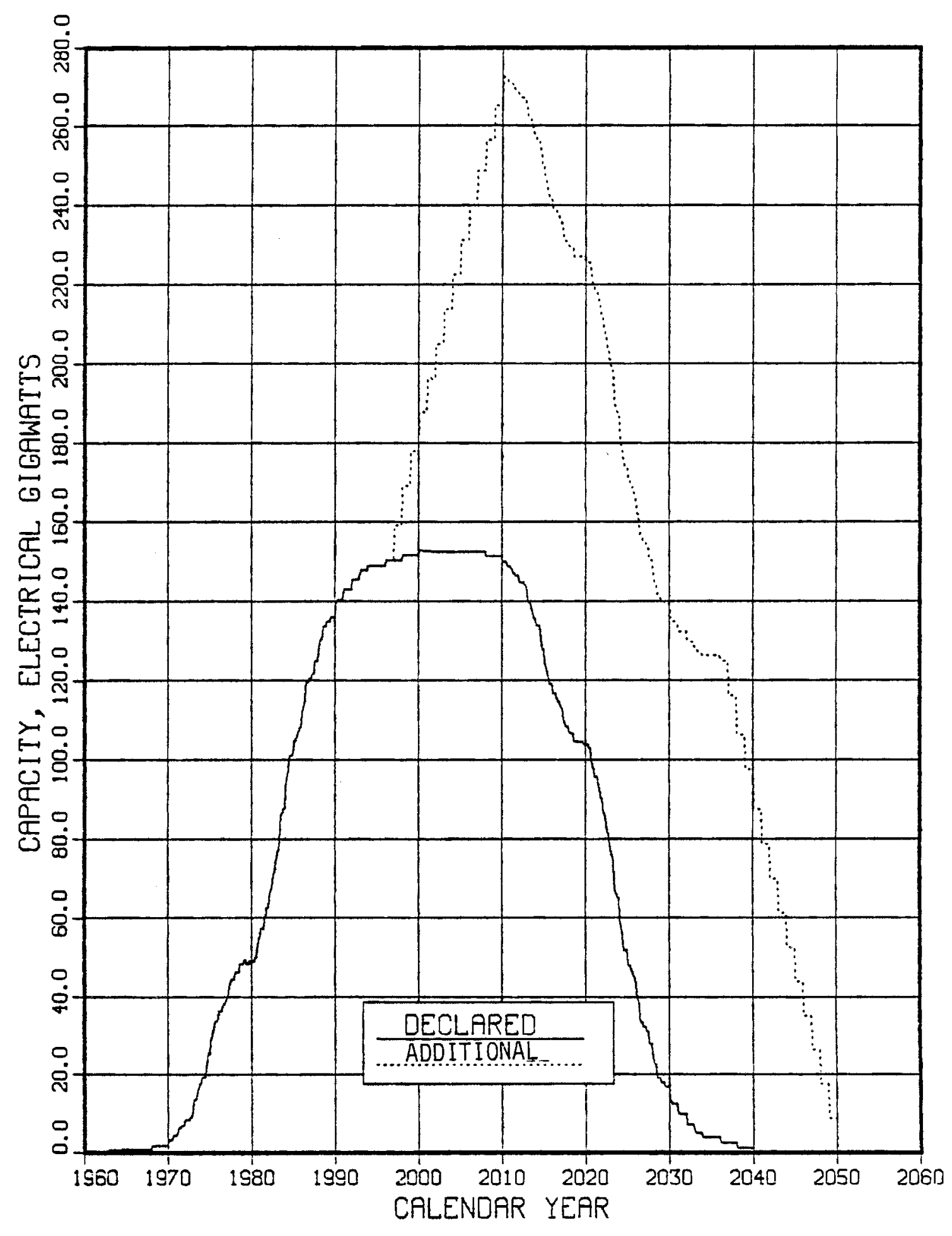

FIGURE 7. U.S. Nuc lear Power - Expanded Scenario Reactor Status 06/15/80 
TABLE 5. Disposal Scenario--Expanded Power Capacity

\begin{tabular}{|c|c|c|c|c|}
\hline $\begin{array}{c}\text { Repository } \\
\text { Identification }\end{array}$ & $\begin{array}{l}\text { Date of } \\
\text { Startup }\end{array}$ & $\begin{array}{l}\text { Date of } \\
\text { Shutdown }\end{array}$ & $\begin{array}{c}\text { Maximum } \\
\text { Loading Rate, } \\
\text { MTU/yr }\end{array}$ & $\begin{array}{c}\text { Repository } \\
\text { Capacity, } \\
\text { MTU }\end{array}$ \\
\hline Rep 1 & 1997.0 & 2032.0 & 2,400 & 84,000 \\
\hline Rep 2 & 2004.0 & 2039.0 & 2,400 & 84,000 \\
\hline Rep 3 & 2023.0 & 2055.0 & 3,000 & 96,000 \\
\hline Rep 4 & 2032.0 & 2060.0 & 3,000 & 84,000 \\
\hline
\end{tabular}




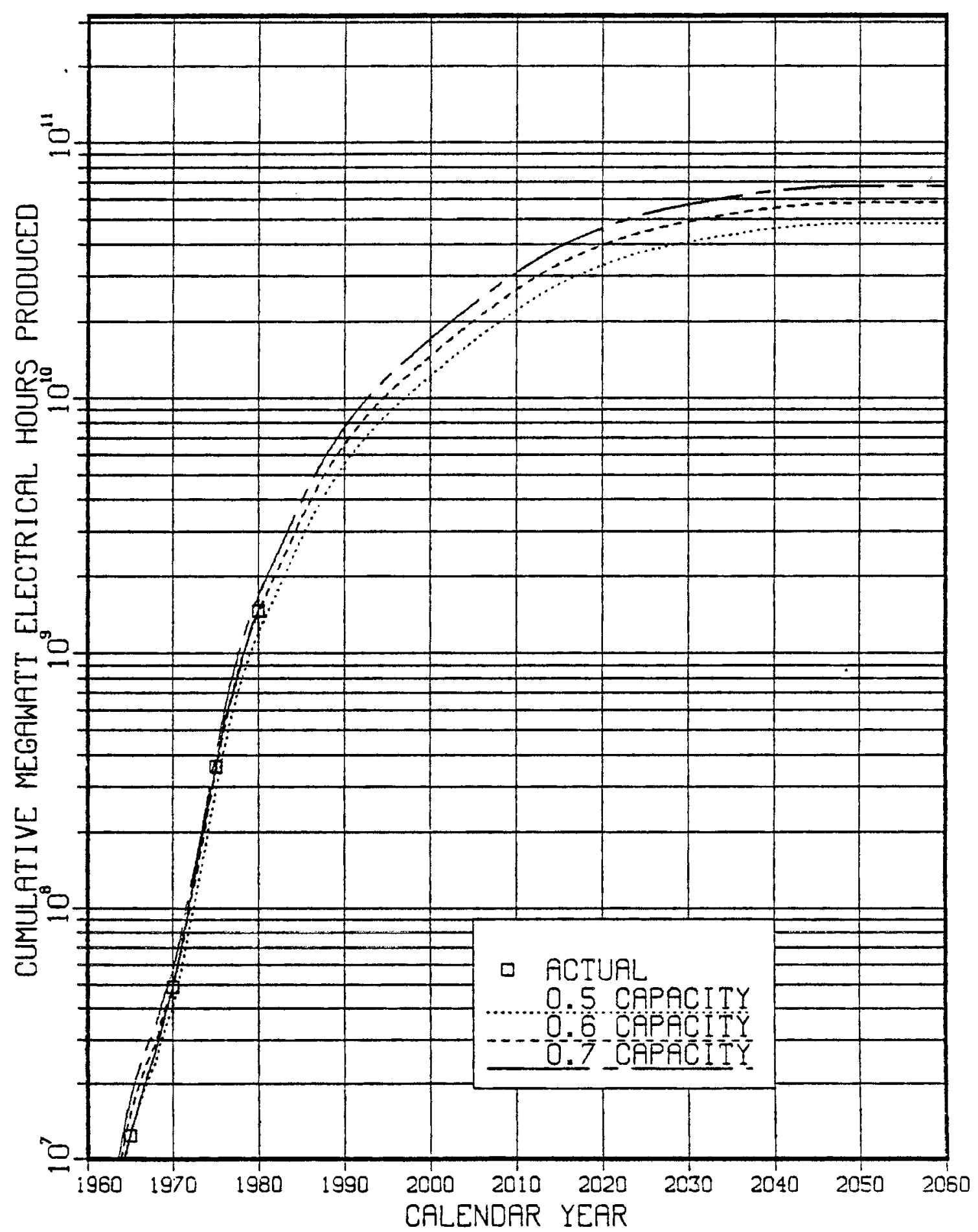

FIGURE 8. U.S. Nuc lear Energy - Expanded Scenario Reactor Status 06/15/80 


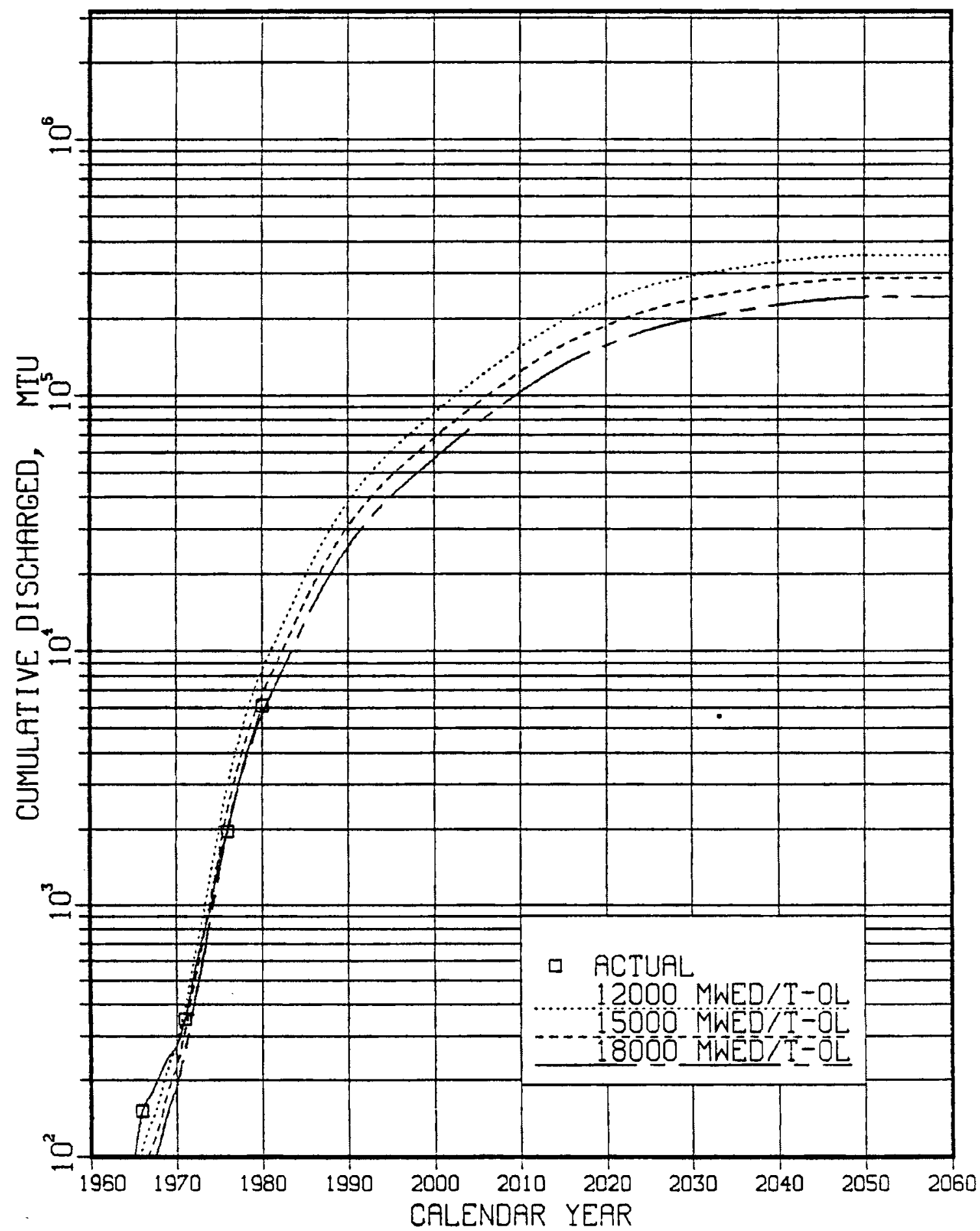

FIGURE 9. U.S. Discharged Fue 1 - Expanded Scenario Reactor Status 06/15/80 


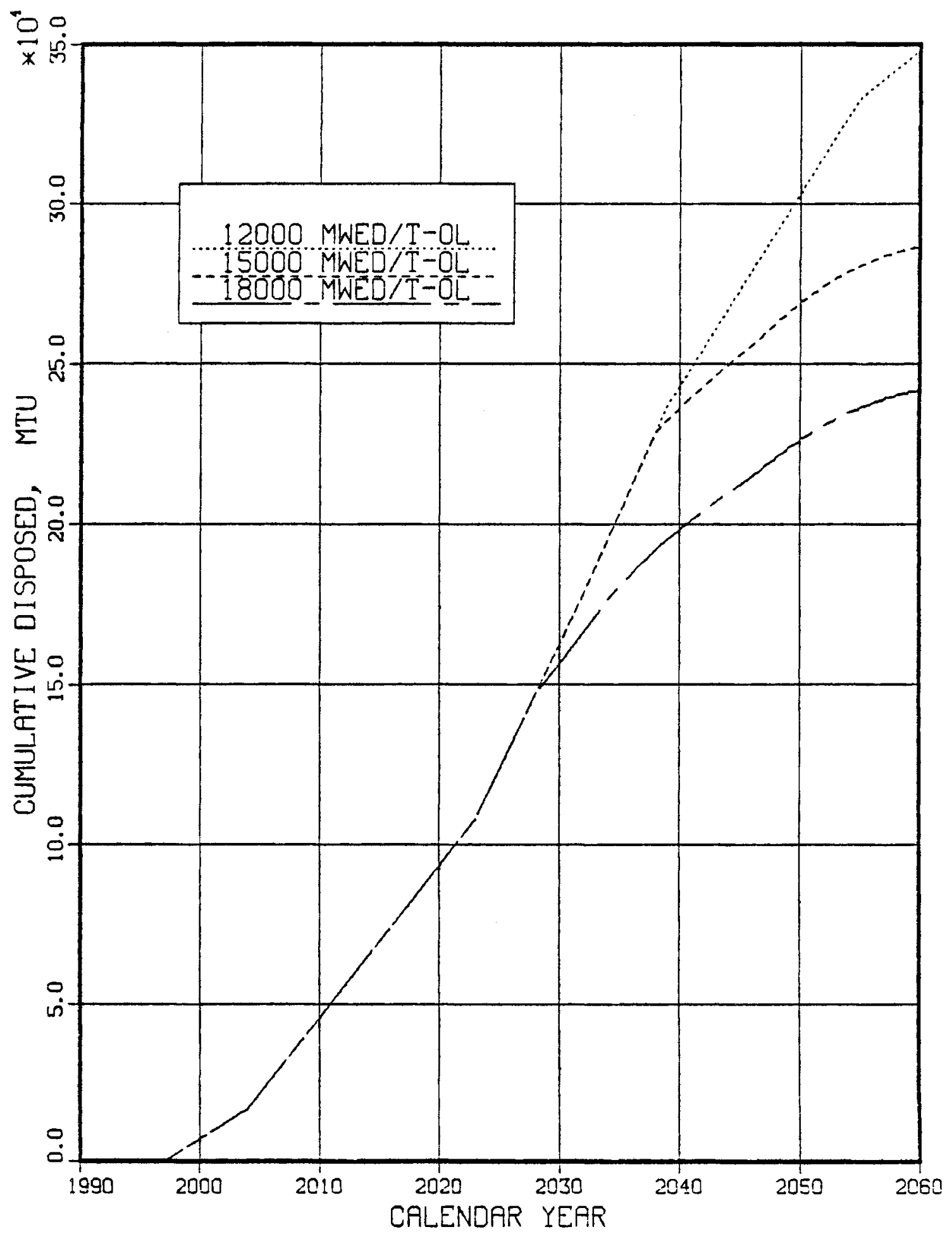

FIGURE 10. U.S. Fuel Disposed - Expanded Scenario Reactor Status 06/15/80

Disposal Start 1997 


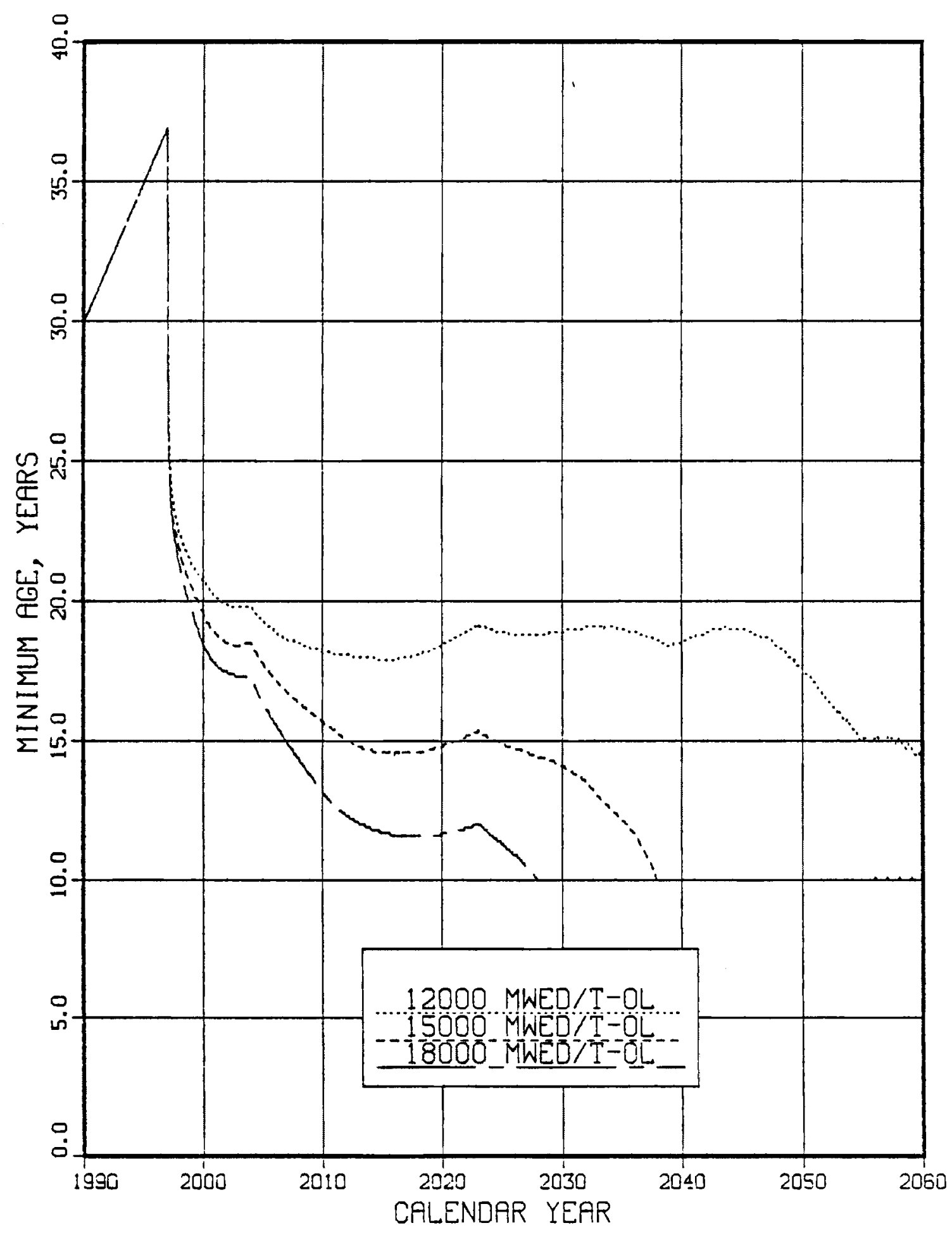

FIGURE 11. Minimum Age of Fue T Disposed - Expanded Scenario Reactor Status 06/15/80 Disposal Start 1997 


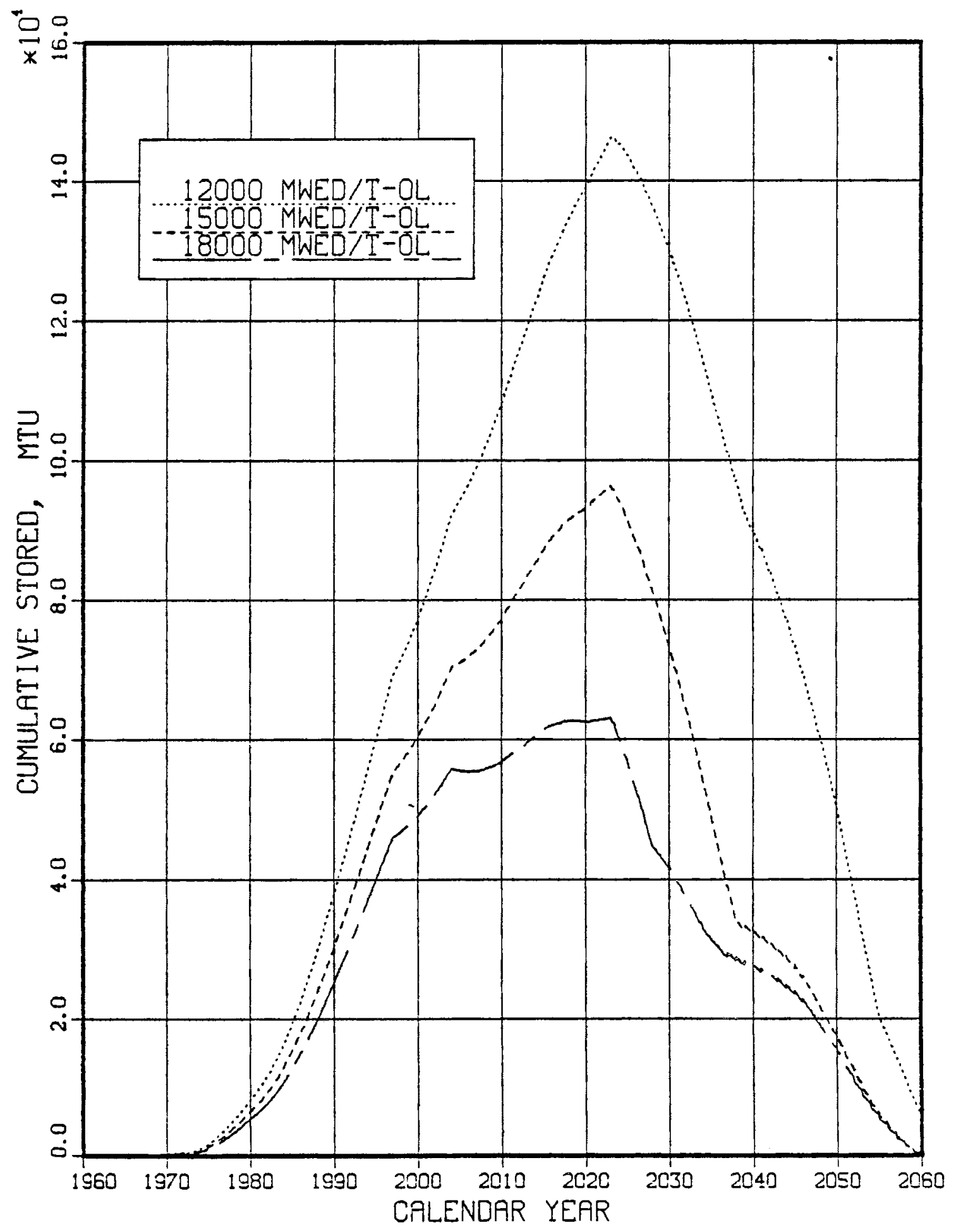

FIGURE 12. Fuel in Predisposal Storage - Expanded Scenario Reactor Status 06/15/80 
TABLE 6. Expanded Nuclear Power Industry

RFACTOR STATIS DG/ISIAR

US R N U C L E A R WOWFA

$G$ in $F$

\begin{tabular}{|c|c|c|}
\hline \multirow[b]{2}{*}{ DATE } & \\
\hline & $\begin{array}{l}\triangle C T U A L \\
\end{array}$ & 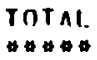 \\
\hline 1961.0 & 0 & $n$ \\
\hline 1962.0 & 0 . & 0 . \\
\hline 1963.0 & 1. & 1. \\
\hline 1964.0 & 1. & 1. \\
\hline 1965.0 & 1. & 1. \\
\hline 1966.0 & 1. & 1. \\
\hline 1967.0 & 1. & 1. \\
\hline 1968.0 & 1. & 1. \\
\hline 1969.0 & 2 . & ?. \\
\hline 1970.0 & 2. & 2. \\
\hline 1971.0 & 4. & 4. \\
\hline 1972.0 & B. & A. \\
\hline 1973.0 & 11. & 11. \\
\hline 1974.0 & $1 A$. & 1A. \\
\hline $1975 . n$ & 25 . & 25. \\
\hline 1976.0 & 35 . & 35. \\
\hline 1977.0 & 39. & 79. \\
\hline 1978.0 & 45 . & 45. \\
\hline 1979.0 & 48. & $4 R$. \\
\hline 1980.0 & 49. & 49. \\
\hline $1981 . n$ & 55. & 55. \\
\hline 19 19.0 & 63. & 6.3. \\
\hline $1983 . n$ & 75. & 75. \\
\hline $19 A 4 . n$ & HA. & AA. \\
\hline 1985.0 & 102. & 1.0?. \\
\hline $198 \% . n$ & 110. & 110 \\
\hline 1987.0 & 121. & 121. \\
\hline 19AR.n & 127. & 127. \\
\hline 19 ค9.0 & 135. & 135. \\
\hline $1990 . n$ & 136. & 136. \\
\hline $\begin{array}{l}1991.0 \\
1992.0\end{array}$ & 142 & 142. \\
\hline $\begin{array}{l}1992.0 \\
1993.0\end{array}$ & $143 \%$ & 143. \\
\hline 1994.0 & 149. & 149. \\
\hline 1995.0 & 149. & 149. \\
\hline 1996.0 & 149. & 149. \\
\hline 1997.0 & 150. & 150. \\
\hline $199 \AA .0$ & 150. & 159. \\
\hline 1999.0 & 152. & 169. \\
\hline
\end{tabular}

NH H A

\begin{tabular}{|c|c|c|}
\hline 5n PER CENT & $\begin{array}{l}\text { 6ก PER CENT } \\
\text { HOS }\end{array}$ & $\begin{array}{l}70 \text { PEP CENT } \\
0\end{array}$ \\
\hline 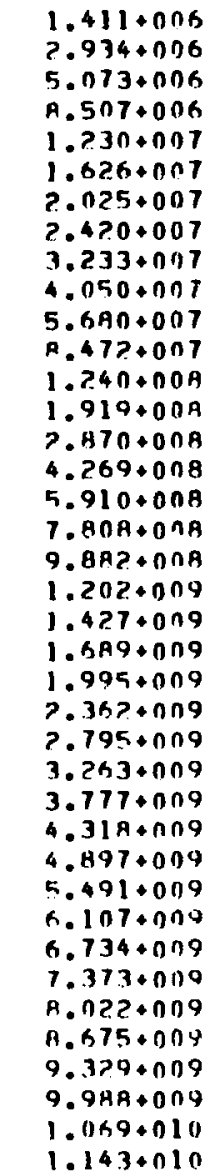 & 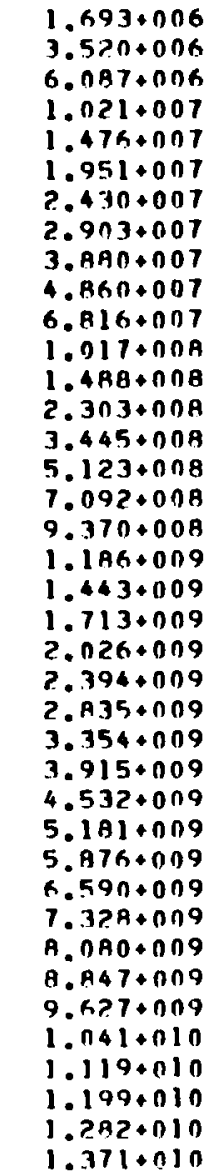 & 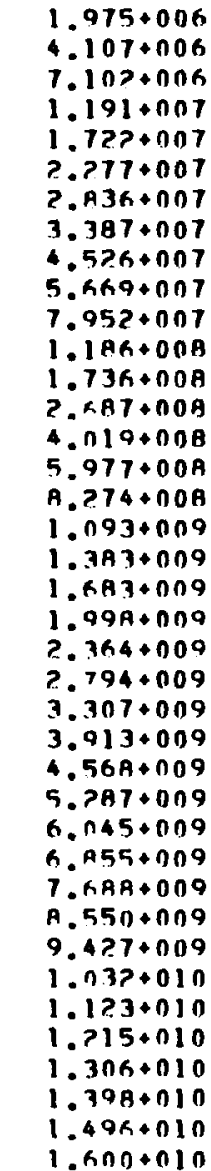 \\
\hline
\end{tabular}

MTU DISCHARGED AT MWFD/T-OI

ACTUAL

a*⿻

$9.720+005$

$2.75 n+0 n 6$

$5.190+006$

a. $140+006$

$1.64 n+007$

?. $160+007$

$2.710+007$

3. 6 . $0+007$

$4.900+007$

$6.960+007$

$1,070+0 \cap 8$

$1,62 n+00 A$

$2.440+00 B$

$3.600+006$

$5.390+008$

$6.910+006$

$1 . ? 10+009$

$1.470+009$

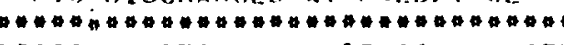

12000 15000 1 AnOD ACTUAL

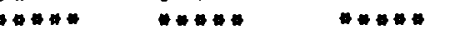

15. $13.0 \quad 30.0$

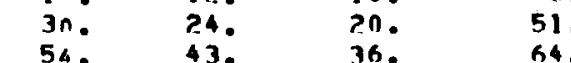

an. 64. 54.56

$10 R$. A6. $12.015 \%$

136. 108. 100.1750

167. 134. 112. ?09.

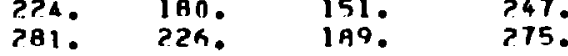

394. 316. 265. 351.

5BA. 47?, 394. $51 \mathrm{~A}$

B61. 690. 576. 731 .

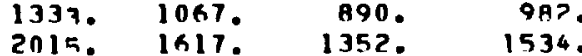

29A6. 2394. 2000 . 1962.

$413 n$. 3310. 2764. 7743.

544A. 4.365. 3643. 3760.

RAAP. 5517. 4602. 4 .

B434. G76R. 5653. 6141.

999R. AD17, 6696.

13047.11173 .0935$.

$13947^{\circ} 11173^{\circ} 9326$.

16493. 13212. 11025.

$19490^{\circ} 15616.1319{ }^{\circ}$

28745. 1AP14. 15194.

26314.21069 .1753$.

30071. 24075. ?007A.

34091. P7>91. 22758.

3A22n, 30594. 25510 .

42495. 34014. 28340.

66A4A. 37496. 31262.

51289. 41047. 34321.

55795. 44654. 37??7.

6037? 4A2A4. 40252.

G4A7I. 51914. 43277.

6944K. 55575. 4637A.

74PAR 59449. 4955K.

7943K. 635K7. 529AB. 
TABLE 6 (contd)

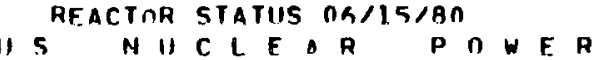

f) " $F$ DATE

औ\#\#

$2000 \cdot 0$

2001.0

20n?

(2003.0

2004.0

2005.0

2006.0

2007.0

$200 \mathrm{~A} .0$

2009.0

2010.0

2011.0

2012.0

2013.0

2014.0

2015.0

2016.0

2018.0

2019.0

2020.0

021.0

2022.0

2023.0

2024.0

2025.0

2026.0

20270

20.31 .0

2032.0

2033.0

2034.0

035.0

2036.0

2037.0

0

2039.0

$$
\text { 1) C LE E R }
$$

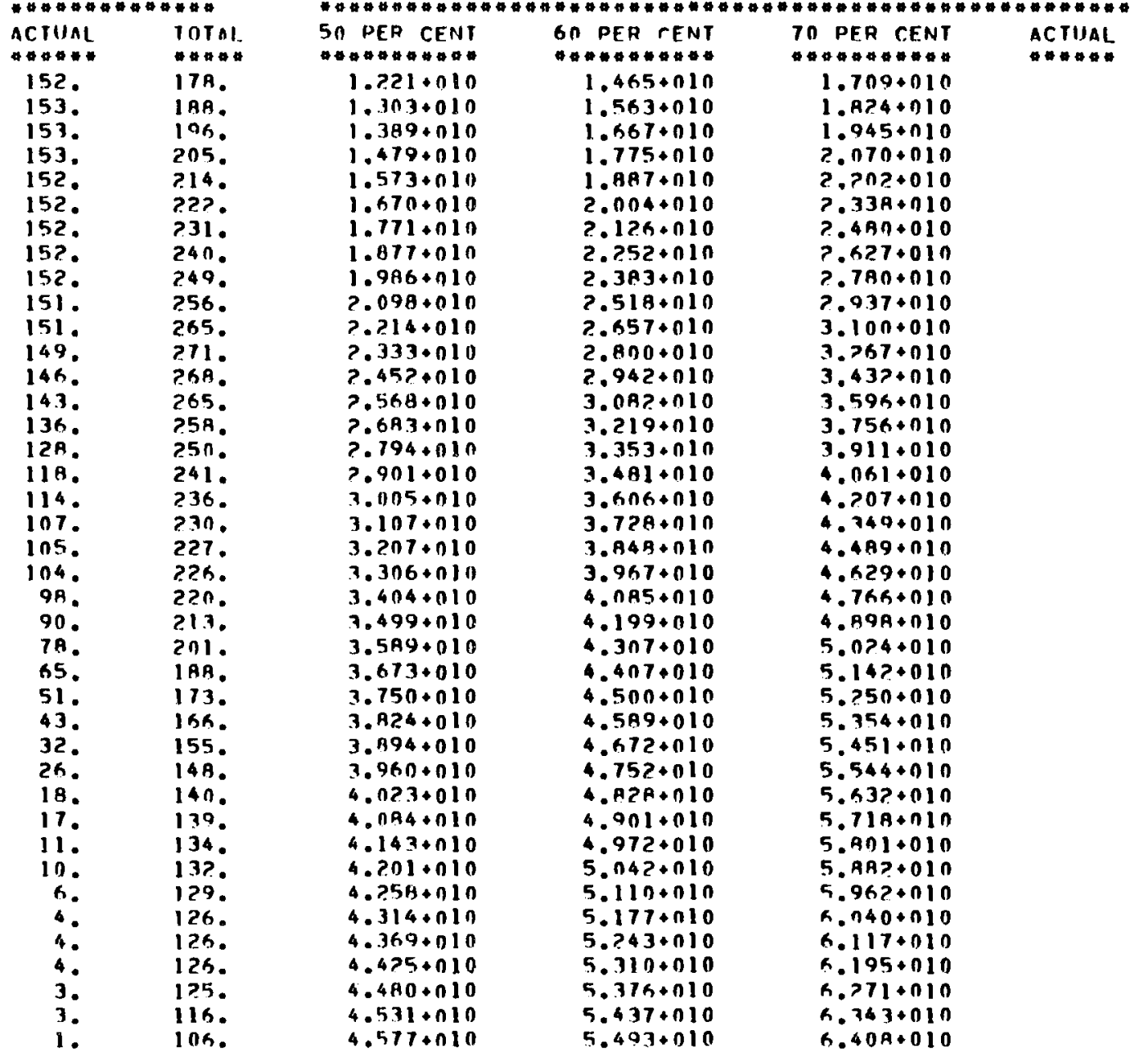

MTU DISCHARGED AT MHED/T-OL

12000 15000

$12000 \quad 15000 \quad 1$ 1AONO ACTUAL

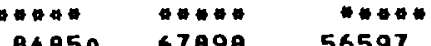

A4B5ก. GTA9R.

905An: 72485, 60421.

9656R. 77278. 64418.

109319. A7479. 72920

109319. A7479. 72920.

$\begin{array}{ll}116090.92896 . & 77474 . \\ 123127.9856 . & 82125\end{array}$

130431.104369 . A6994.

138064 . 110492 . 92108 .

145A74. 116737. 97312.

154037. 1232A2. 1027A1.

16243?. 130024. 108419.

170A6R. 136819. 1141?0.

179.327. 143655. 119874.

$1 A 7637.1503 A 0$. 125542

$196004 \cdot 1572 n 0.131337$.

203931. 16.364k. 136788.

11425. 16969. 1414Ke。

21A91a. 17576A. 147003.

22599?. 1A145K, 151765.

?32949.18703?. 1564?1.

240320.193039 .161519$.

247395. 199400, 166404 .

254445. 204601. 171371.

261356. 210343 . 176336.

267523. 215436. lan711.

27311R. ?PND04. 1A4594.

?7A52n. 224443. 18839?

2A36Bn.

(3)

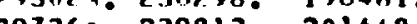

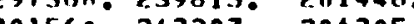

$30156 n$. 243207. $2043 n 5$.

3n5khs: 246521: 207093.

$309 \mathrm{kln}$. 249694. 209750

313455 . 25277n. ?12313.

1173Ak. 255932. 214962

$32177 \mathrm{k}$. ?59560. 2IAnA3.

3259A5. 263061. 221112 
TABLE 6 (contd)

REACTIRR STATUS OG/15/BN

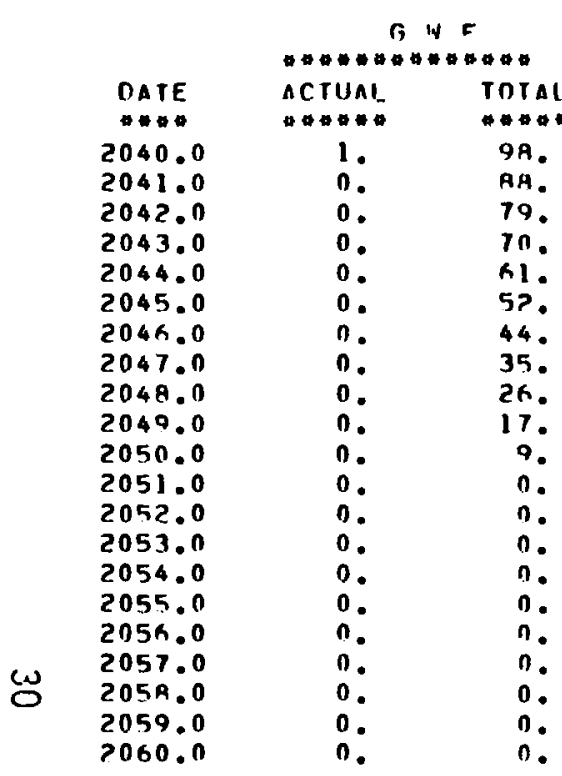

11 S N ICLEAR

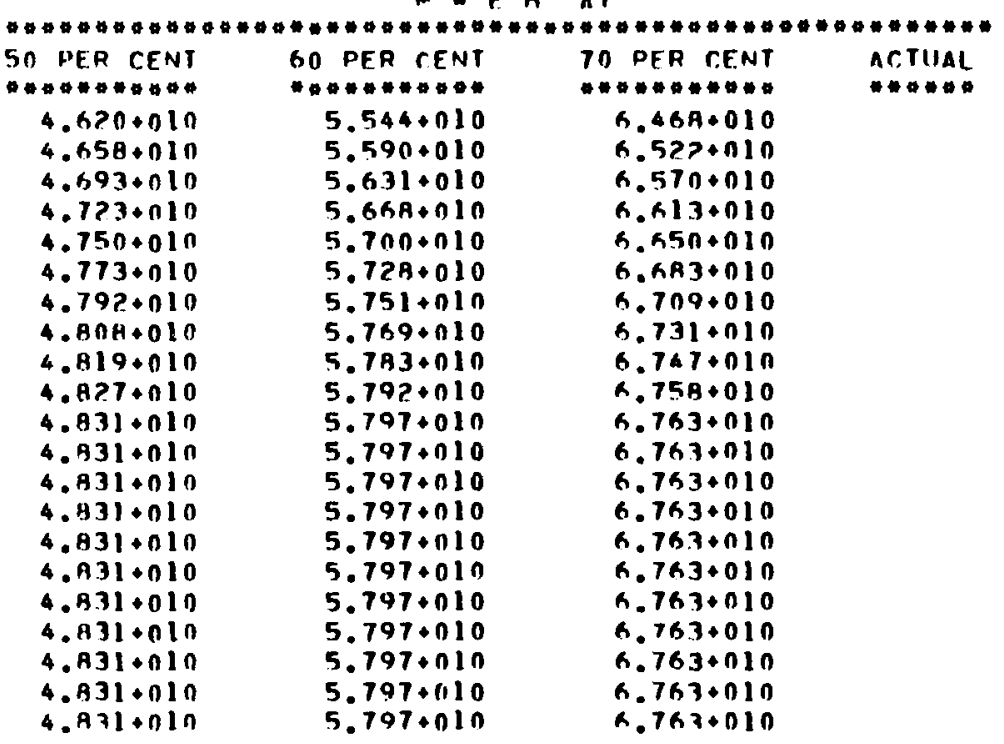

MIII DISCHARGED AT MWED/T-OI

\begin{tabular}{|c|c|c|c|}
\hline 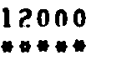 & $\begin{array}{l}15000 \\
6 * \ldots *\end{array}$ & $\begin{array}{r}18000 \\
* * * *\end{array}$ & $\begin{array}{l}\text { ACTUAL } \\
\text { W }\end{array}$ \\
\hline 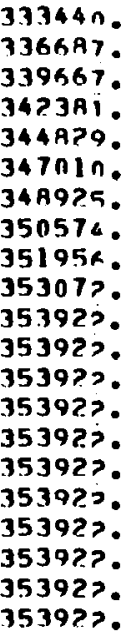 & 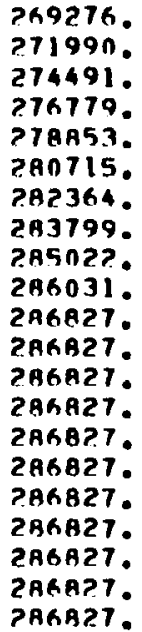 & 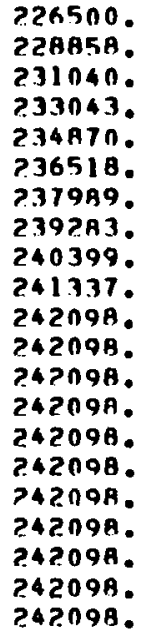 & \\
\hline
\end{tabular}


TABLE 6 (contd)

RFACINR STATIJS OG/15/AO

FUF, STATISS. MTU

15,000 MHEN/T-OL SCEMARIO

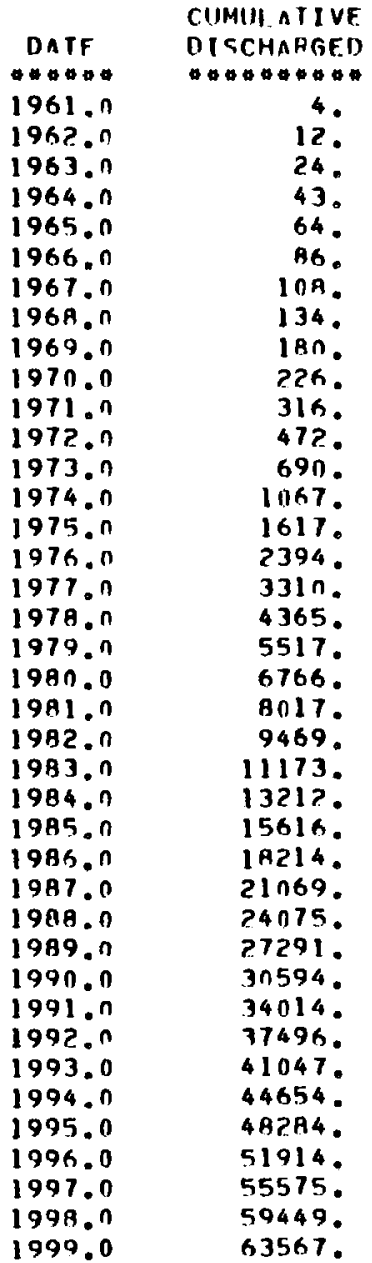

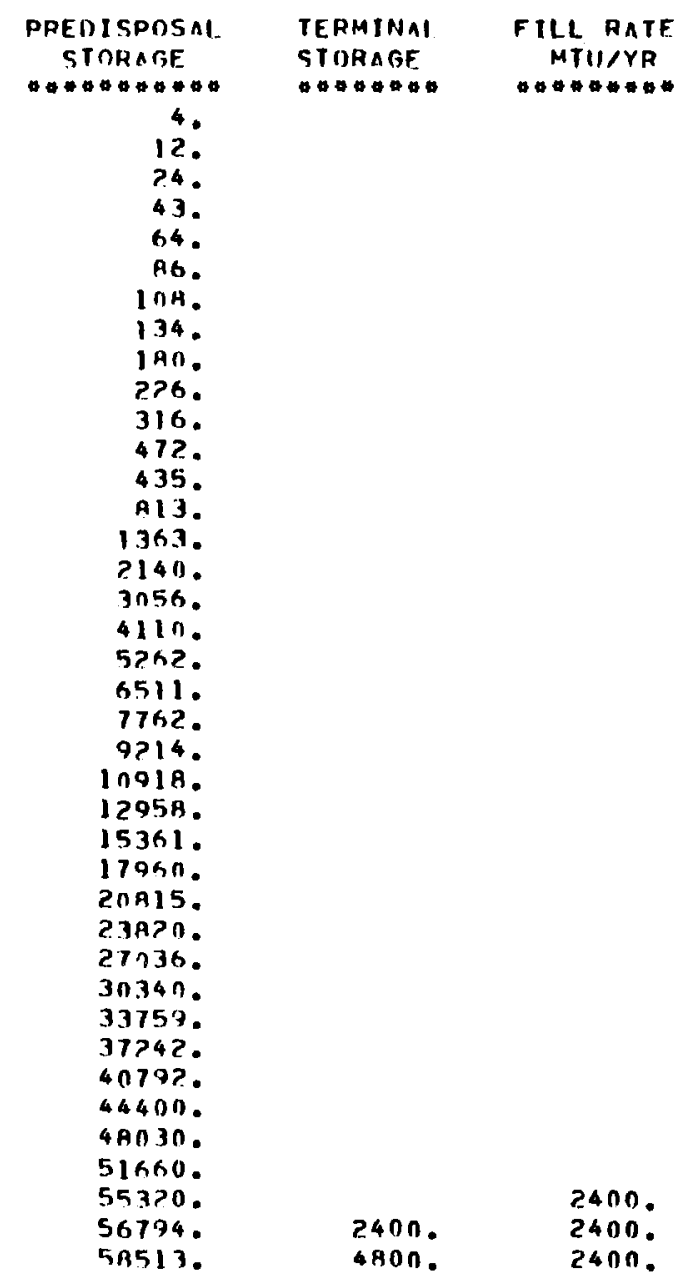

\begin{tabular}{|c|c|}
\hline $\begin{array}{l}\text { CAPACITY } \\
\text { MTIIYYR } \\
\text { W }\end{array}$ & $\begin{array}{r}\text { MINIMUM } \\
\text { AGE YYS } \\
\text { H. } \\
.9 \\
1.9 \\
7.9 \\
3.9 \\
4.9 \\
5.9 \\
6.9 \\
7.9 \\
8.9 \\
9.9 \\
11.9 \\
11.9 \\
12.9 \\
13.9 \\
14.9 \\
15.9 \\
16.9 \\
17.9 \\
18.9 \\
19.9 \\
20.9 \\
21.9 \\
22.9 \\
23.9 \\
24.9 \\
25.9 \\
26.9 \\
27.9 \\
28.9 \\
29.9 \\
30.9 \\
31.9 \\
32.9 \\
33.9 \\
34.9 \\
35.9\end{array}$ \\
\hline $\begin{array}{l}2400 . \\
2400 . \\
2400 .\end{array}$ & $\begin{array}{l}36.9 \\
21.9 \\
20.6\end{array}$ \\
\hline
\end{tabular}


$\underline{\text { TABLE } 6}$ (contd)

REACTAR STATISS NG/15/AR

FUFI STATUS. MTU

15,000 MWED/T-OL SCENARIO

\begin{tabular}{|c|c|}
\hline \multirow{2}{*}{ DATE } & $\begin{array}{l}\text { CIIMUL.ATIVE } \\
\text { DISCHARGED }\end{array}$ \\
\hline & 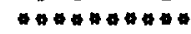 \\
\hline & 67898 \\
\hline 2001.0 & 72485 . \\
\hline 2002.0 & $7727 \mathrm{~A}$. \\
\hline 2003.0 & 82270 . \\
\hline 2004.0 & 87479 . \\
\hline 2005.0 & 92896. \\
\hline 2006.0 & 9A526. \\
\hline $2007 . n$ & 104369. \\
\hline 200 A. 0 & 110492. \\
\hline 2009.0 & 116737. \\
\hline 2010.0 & 123282. \\
\hline 2011.0 & $130 n 24$. \\
\hline 2012.0 & 136819 \\
\hline 2013.0 & 143655 . \\
\hline 2014.0 & 150380. \\
\hline 2015.0 & 157200. \\
\hline 2016.0 & 163646. \\
\hline 2017.0 & 169691. \\
\hline 201 A. 0 & $17576 \mathrm{~A}$. \\
\hline 2019.0 & 181456 . \\
\hline 2020.0 & 187n3?. \\
\hline 2021.0 & 193039 . \\
\hline 2022.0 & 198800. \\
\hline 2023.0 & 204601. \\
\hline 2024.0 & 210343. \\
\hline 2025.0 & 215436. \\
\hline 2026.0 & 220004. \\
\hline 2027.0 & 224443. \\
\hline $202 \mathrm{R} .0$ & $22 A 658$. \\
\hline 2029.0 & 232591 . \\
\hline 20.30 .0 & 23629A. \\
\hline 2031.0 & 239813. \\
\hline 20.32 .0 & 2.3207. \\
\hline 2033.0 & 246521 . \\
\hline 2034.0 & 249694 . \\
\hline 2035.0 & $25277 n$. \\
\hline 2036.0 & 255932 . \\
\hline 2037.0 & 259560. \\
\hline $203 R_{0} n$ & 26ankl. \\
\hline 2039.0 & 26K23?. \\
\hline
\end{tabular}

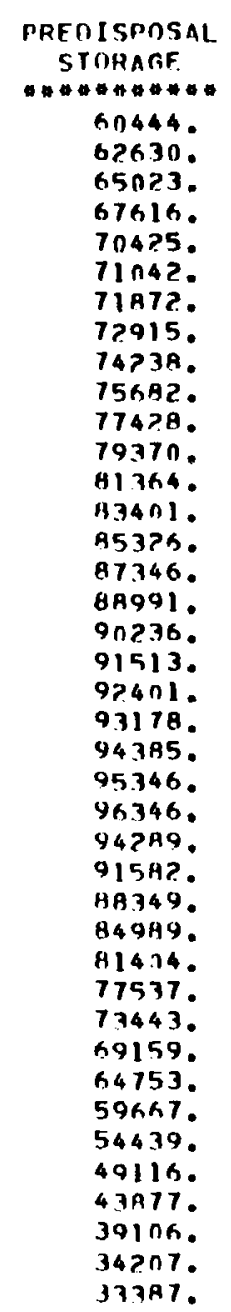

TERMINAL

STORAGF.

*a*a***

7200.

12000.

12000 .

14400.

16800 .

21600.

26400 .

$312 n 0$.

36000 .

$40 \mathrm{000}$.

50400 .

55200 .

60000.

60nก)

64800.

74400 .

74400.

79200 .

B4กNO

PBANO.

98400 .

103200 .

108000 .

II5RUn.

123600 .

123600

139200.

147000 .

147000.
$154 A B D$.

162600 .

170400 .

170400.
178200

I BGSOO.

195000

20.3400

211 A0N.

2?0?00.

228600.

232591 .

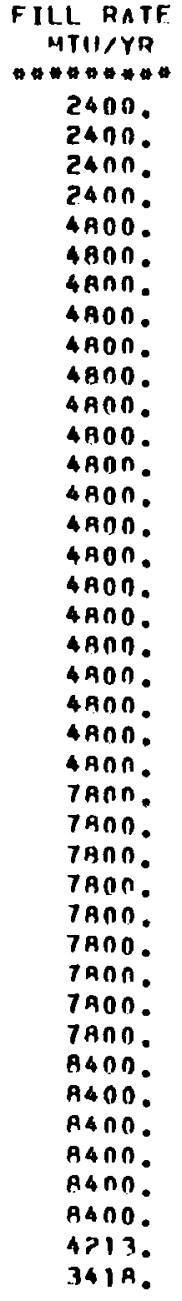

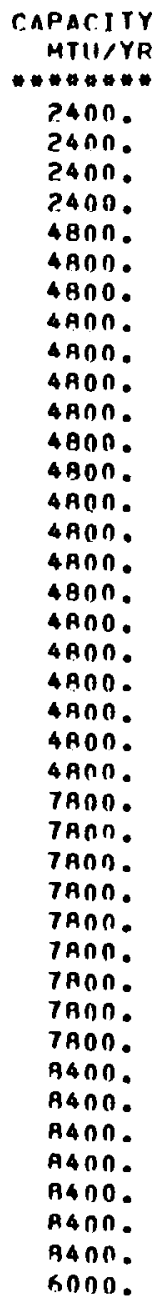

MINIMUM

AGE, YAS

* * * *

19.6

18.9
18.5

18.5

18.5

17.8

17.2

16.8

16.4

16.4

16.0
15.7

15.4

15.1

15.1

14.8

14.6

14.6
14.5

14.5
14.6

14.6
14.6

14.6
14.7

14.8

15.0

$15 . ?$

15.4

15.1

14.9

14.9

14.6

14.4

14.3

14.1

13.8

13.5

13.0

12.6

12.2

11.7

10.0

GODO.

10.0 
TABLE 6 (contd)

REACINH STATISS DG/15/AO

FUFI STATUS. MTII

15,000 MWEO/T-OL SCENARIO

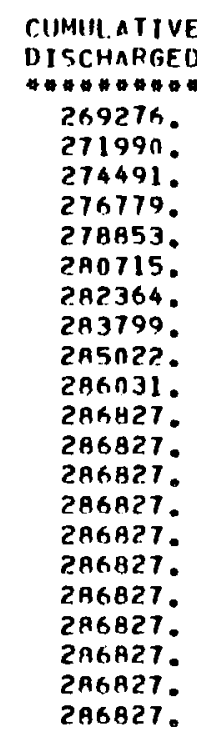

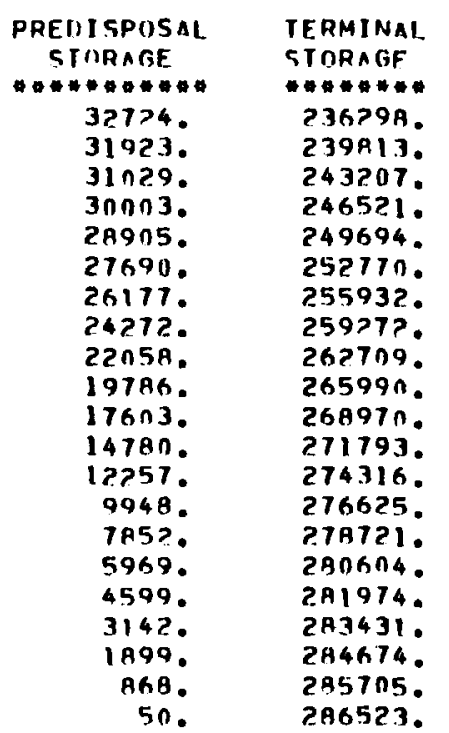

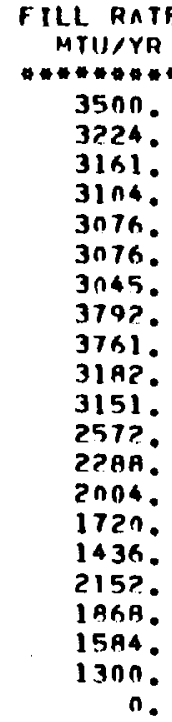

$\begin{array}{cc}\text { CAPACITY } & \text { MINIMUM } \\ \text { MTUYY } & \text { GE YYRS } \\ \text { GOON. } & 10.0 \\ 6000 . & 10.0 \\ 6000 . & 10.0 \\ 6000 . & 10.0 \\ \text { GOON. } & 10.0 \\ 6000 . & 10.0 \\ 6000 . & 10.0 \\ 6000 . & 10.0 \\ \text { 6000. } & 10.0 \\ 6000 . & 10.0 \\ 6000 . & 10.0 \\ 6000 . & 10.0 \\ 6000 . & 10.0 \\ 6000 . & 10.0 \\ 6000 . & 10.0 \\ 6000 . & 10.0 \\ 3000 . & 10.0 \\ 3000 . & 10.0 \\ 3000 . & 10.0 \\ 3000 . & 10.0 \\ 3000 . & 10.0 \\ 0 . & \end{array}$


Table 7. Comparison of the Two Power Scenarios

Declared Plus

Declared Reactors

Peak capacity, GWe

Energy, MWEH

Fuel discharged, MTU

Maximum fuel in predisposa1, MTU

Maximum total repository load rate, $M T U / y r$

Enrichment feed, MTU
153

$3.22+010$

159,000

82,000 (in 2020)

5,000

872,000
Additional Reactors

273

$5.80+010$

287,000

96,000 (in 2023)

8,400

$1,570,000$ 


\section{REFERENCES}

1. United States Department of Energy, U.S. Central Station Nuclear Electric Generating Units: Significant Milestones, DOE/NE-0030/2(80), June 1980.

2. Nuclear Assurance Corporation, U.S. LWR Spent Fuel Inventory and Projection, Y/OWI/SUB-77/42500, June 1977.

3. C. W. Alexander, J. 0. Blomeke et al., Projections of Spent Fuel to be Discharged by the U.S. Nuc lear Power Industry, ORNL/TM-6008, Oak Ridge National Laboratory, October 1977.

4. United States Nuclear Regulatory Commission, Operating Units Status Report, Data as of 10-31-79; Licensed Operating Reactors, Data for Decisions, NUREG-0020, Vol. 3, No. 11, November 1979.

5. United States Department of Energy, Draft Environmental Impact Statement: Management of Commercially Generated Radioactive Waste, DOE/EIS-0046-D, Volumes 1 and 2, ApriT 1979.

6. United States Department of Energy, Technology for Commercial Radioactive Waste Management, DOE/ET-0028, Volumes 1 through 5, May 1979.

7. R. I. Smith, G. J. Konzek, and W. E. Kennedy, Jr., Technology, Safety, and Costs of Decommissioning a Reference Pressurized Water Reactor Power Station, NUREG/CR-0130, Vol. 2, Appendix G, June 1978.

8. United States Nuclear Regulatory Commission, Environmental Survey of the Reprocessing and Waste Management Portions of the LWR Fuel Cycle, A Task Force Report, NUREG-0116, October 1976.

9. International Nuclear Fuel Cycle Evaluation, Waste Arisings from Reactor and Post-Fission Activities in Selected Fuel Cycles, Report by Working Group 7, INFCE/DEP/WG.7/11, Vienna, August 1979.

10. International Nuclear Fuel Cycle Evaluation, Waste Management and Disposal for Selected Nuclear Fuel Cycles, Report by working Group 7, INFCE/WG.7/26, Vienna, May 1979. 
.

-

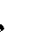




\section{APPENDIX}

DETAILED INFORMATION ON LWRS

INCLUDED IN AND EXCLUDED FROM THE ANALYSES 
TABLE A-1. U.S. LWR Commercial Reactors - PWR

ON-LINE/DOWN

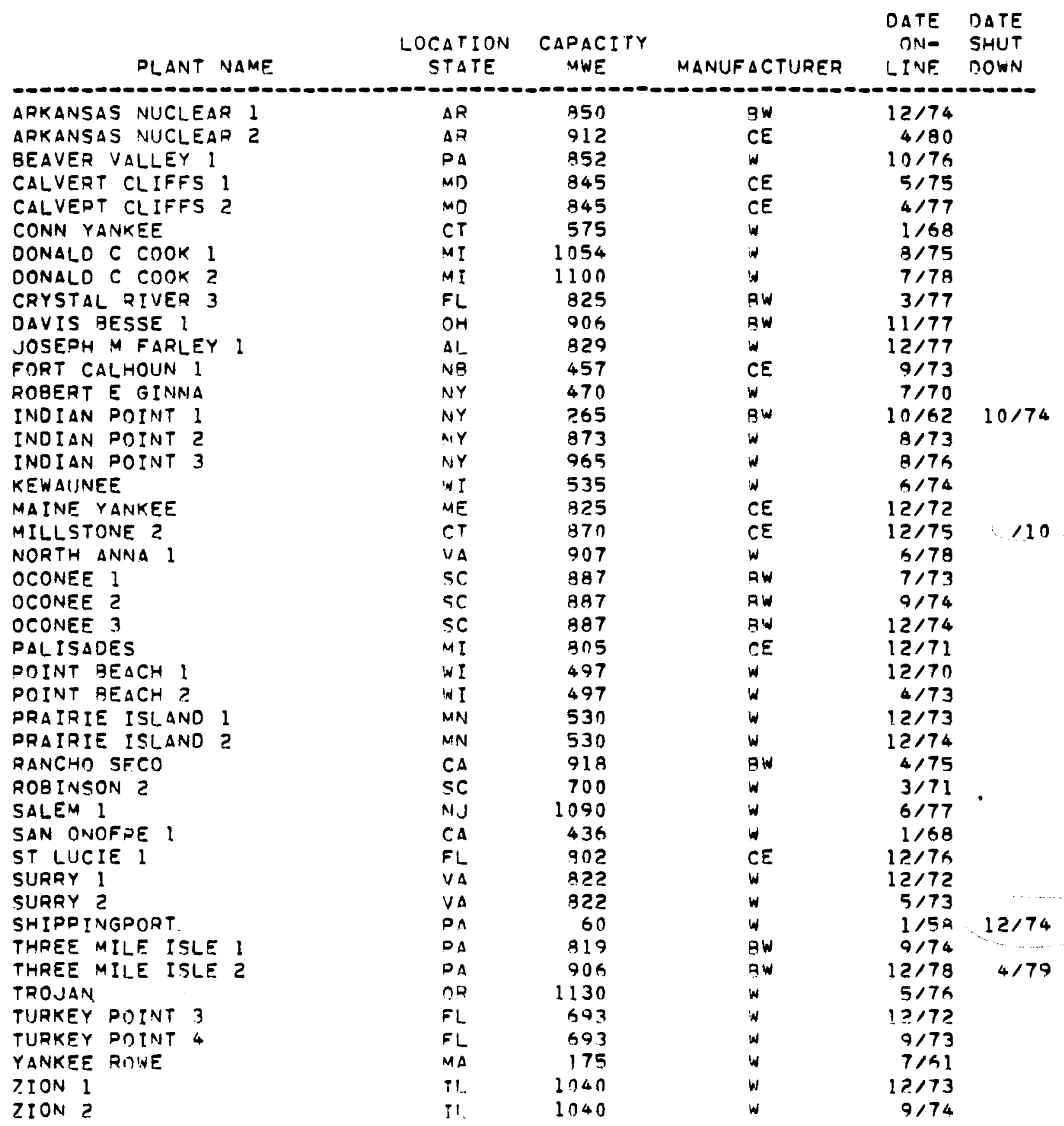


TABLE A-2. U.S. LWR Commercial Reactors - PWR

PRDJECTED

\begin{tabular}{|c|c|c|c|c|}
\hline Plant name & $\begin{array}{l}\text { LOCATION } \\
\text { STATE }\end{array}$ & $\begin{array}{l}\text { CAPACITY } \\
\text { MWE }\end{array}$ & MANIJFACTURER & $\begin{array}{c}\text { D_ANNED } \\
\text { DATE } \\
\text { DNE } \\
\text { LINE }\end{array}$ \\
\hline ALLENS CZEEK l & $T x$ & 1150 & GE & $11 / 87$ \\
\hline BEAVER VALLEY 2 & $P_{A}$ & 8.3 .3 & $w$ & $5 / 86$ \\
\hline BELLEFONTE 1 & $\Delta L$ & $1 \geq 13$ & Aw & $0 / 83$ \\
\hline RELLEFONTE ? & $\Delta \mathrm{I}_{-}$ & 1213 & $9 \mathrm{~N}$ & $6 / 84$ \\
\hline RRAIDWOOD I & IL & 1120 & $w$ & $0 / 85 *$ \\
\hline BRAIOWOOD Z & IL & 1120 & $w$ & $0 / 86 *$ \\
\hline BYRON 1 & IL & 1120 & $w$ & $0 / 83 *$ \\
\hline BYRON? & IL & 1120 & $w$ & $0 / 84 *$ \\
\hline CALLAWAY 1 & $M D$ & $112 n$ & $w$ & $10 / 82$ \\
\hline CALLAWAY? & MO & 1120 & $w$ & $0 / 88 *$ \\
\hline CARROLL 1 & IL & 1120 & $w$ & $10 / 92$ \\
\hline CARROLL ? & $\mathrm{PI}$ & 1120 & $w$ & $10 / 93$ \\
\hline CATAWBA 1 & sc & 1145 & $\omega$ & $3 / 84 *$ \\
\hline CATAWAA? & $s c$ & 1145 & $\omega$ & $9 / 85 *$ \\
\hline CHEROKEE 1 & SC & 1280 & $C E$ & $1 / 90 *$ \\
\hline CHEROKEE 2 & $s c$ & 1280 & $C E$ & $1 / 92$ * \\
\hline COMANCHE PEAK 1 & $T x$ & 1111 & $w$ & $12 / 21$ \\
\hline COMANCHE PEAK 2 & $T X$ & 1111 & $w$ & $12 / 83$ \\
\hline DI ABLO CANYON 1 & CA & 1084 & $\mathbf{w}$ & $0 / 91 *$ \\
\hline DIABLO CANYON $?$ & $\mathrm{CA}$ & 1106 & $w$ & $0 / 81$ \\
\hline JOSEPH M FARLEY 2 & $A L$ & 829 & w & $9 / 80$ \\
\hline MARBLE HILL I & IN & 1130 & $w$ & $10 / 82$ \\
\hline MARBLE HILL? & IN & 1130 & $w$ & $1 / 84$ \\
\hline MCGUIRE I & NC & 1180 & $w$ & $9 / 8 O$ \\
\hline MCGUIAE 2 & NC & 1180 & $w$ & $9 / 82 *$ \\
\hline MIDLAND I & MI & 460 & AW & ก/85* \\
\hline MIDLAND 2 & is I & 811 & BW & $12 / 83$ \\
\hline MILLSTONE 3 & $C T$ & 1156 & $w$ & $5 / 86$ \\
\hline NEW HAVEN I & NY & $1 \geq 50$ & CE & $12 / 92$ \\
\hline NORTH ANNA $Z$ & VA & 907 & $w$ & $8 / 80 *$ \\
\hline PALO VERDE I & $\Delta 7$ & 1270 & $C E$ & $5 / 83$ \\
\hline PALO VERDE 2 & A 7 . & 1270 & CE & $5 / 84$ \\
\hline PALO VERDE 3 & $A Z$ & 1270 & $C E$ & $6 / 86$ \\
\hline PEBALE SPRINGS 1 & $O R$ & 1260 & QW & $11 / 88$ \\
\hline DEBBLE SDRINGS? & $O R$ & 1280 & SW & $11 / 90$ \\
\hline PERKINS 1 & NC & 1280 & CE & $1 / 96 *$ \\
\hline PERKINS? & NC & 1280 & CE & $1 / 98 *$ \\
\hline PERKINS 3 & NC & 1280 & CE & $1 / 0 *$ \\
\hline PILGRIM 2 & MA & 1150 & $C E$ & $12 / 85$ \\
\hline ST LUCIE 2 & FL & 310 & CE & $5 / 83$ \\
\hline SALEM 2 & $\mathrm{NJ}$ & 1115 & $w$ & $10 / 80$ \\
\hline SAN ONOFRE 2 & CA & 1100 & CE & $12 / 81 *$ \\
\hline SAN ONOFRE 3 & $\mathrm{CA}$ & 1100 & $C E$ & $1 / 83$ \\
\hline
\end{tabular}


TABLE A-2 (contd)

PROJECTEO

\begin{tabular}{|c|c|c|c|c|c|}
\hline & PLANT NAME & $\begin{array}{l}\text { LOCATION } \\
\text { STATE }\end{array}$ & $\begin{array}{l}\text { CAPACITY } \\
\text { MWE }\end{array}$ & MANUFACTIIRER & $\begin{array}{l}\triangle N N E D \\
\text { DATE } \\
\text { ONE } \\
\text { LTNE }\end{array}$ \\
\hline$-\infty-\infty-\infty$ & $--\infty-\infty-\infty-\infty$ & - & - - - - - - - & 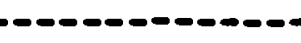 & $-\infty-\infty$ \\
\hline SEABROOK & 1 & $\mathrm{NH}$ & 1200 & $w$ & $4 / 83$ \\
\hline SEABROOK & 2 & $\mathrm{NH}$ & 1200 & $w$ & $2 / 88 *$ \\
\hline SEQUOYAH & 1 & $T N$ & 1148 & $w$ & $9 / 80 *$ \\
\hline SEQUOYAH & 42 & TN & 1148 & $w$ & $6 / 81$ \\
\hline SHEARON & HARRIS 1 & $N C$ & 900 & $w$ & $3 / 84$ \\
\hline SHEARON & HARRIS 2 & NC & 900 & $w$ & $3 / 86$ \\
\hline SHEARON & HARRIS 3 & NC & 900 & w & $3 / 90$ \\
\hline SHEARON H & HARRIS 4 & NC & 900 & $w$ & $3 / 88$ \\
\hline SO TEXAS & DROJECT 1 & $T X$ & 1250 & $w$ & $2 / 84$ \\
\hline SO TEXAS & PROJECT 2 & $T X$ & 1250 & $w$ & $2 / 86$ \\
\hline VANOALIA & NUCLEAR & I A & 1270 & RW & $12 / 90$ \\
\hline VIRGIL C & SUMMER 1 & sc & 900 & W & 8/81* \\
\hline VOGTLE 1 & & GA & 1110 & $\omega$ & $11 / 84$ \\
\hline VOGTLE 2 & & GA & 1110 & $w$ & $11 / 87$ \\
\hline WPPSS 1 & & WA & 1218 & BW & $12 / 83$ \\
\hline WPPSS 3 & 8 & WA & 1242 & CE & $12 / 84$ \\
\hline WPPSS 4 & “ & WA & 1218 & $B w$ & $6 / 87 *$ \\
\hline WPPSS 5 & & WA & 1240 & CE & $5 / 87 *$ \\
\hline WOLF CREE & EEK & K.5 & 1150 & $w$ & $4 / 83$ \\
\hline WATERFORL & 203 & LA & 1113 & $C E$ & $2 / 82$ \\
\hline WATTS BAE & A 1 & $T y$ & 1177 & $w$ & $9 / 81$ \\
\hline WATTS BAF & $R 2$ & Tis & 1177 & $w$ & $6 / 82$ \\
\hline YELLOW CF & SREEK 1 & MS & 1285 & $C E$ & $11 / 85$ \\
\hline
\end{tabular}

* Date is later than reported in PNL-3317-1. 
TABLE A-3. U.S. LWR Commercial Reactors - PWR

CANCELLED

\begin{tabular}{|c|c|c|c|c|}
\hline PLANT NAME & $\begin{array}{c}\text { LOCATION } \\
\text { STATE }\end{array}$ & $\begin{array}{c}\text { CAPACITY } \\
\text { AWE }\end{array}$ & MANUF ACTURER & $\begin{array}{c}\text { LANNED } \\
\text { DATE } \\
\text { ONE } \\
\text { LINF }\end{array}$ \\
\hline ATLANTIC 1 & $.0-$ & - & 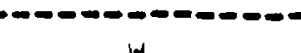 & ----- \\
\hline $\begin{array}{l}\text { ATLANTIC ! } \\
\text { ATLANTIC? }\end{array}$ & $\begin{array}{l}\mathrm{NJ} \\
\mathrm{NJ}\end{array}$ & $\begin{array}{l}1150 \\
1150\end{array}$ & $w$ & $\begin{array}{l}5 / 85 \\
5 / 87\end{array}$ \\
\hline 1990 UNNIT & AIY & 1150 & $\omega$ & $5 / 90$ \\
\hline 1992 UNIT & NY & 1150 & $\omega$ & $5 / 9 ?$ \\
\hline BLUE HILLS ? & $T x$ & 918 & $c$ & $n / 89$ \\
\hline BLUE HILLS 2 & $T X$ & 918 & $C E$ & $0 / 91$ \\
\hline CAROLINA P-L \& & sc & 1150 & Bw & $3 / 87$ \\
\hline CAROLINA P-L 9 & NC & 1150 & RW & $3 / 89$ \\
\hline CAROLINA P-L I & NC & 1150 & BW & $3 / 91$ \\
\hline DAVIS BESSE ? & $\mathrm{OH}$ & 906 & SW & $12 / 88$ \\
\hline DAVIS BESSE 3 & $\mathrm{OH}$ & 906 & $B w$ & $12 / 90$ \\
\hline ERIE I & OH & 1260 & Rw & $4 / 86$ \\
\hline ERIE 2 & 04 & 1260 & BW & $4 / 88$ \\
\hline FT CALHOUN 2 & NB & 1136 & $w$ & $1 / 83$ \\
\hline GREENE CO & NY & 1191 & RW & $9 / A 4$ \\
\hline GREENWOON ? & 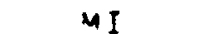 & 12.64 & $8 w$ & $9 / 90$ \\
\hline GREENWOOD 3 & $M I$ & 1200 & RW & $919 ?$ \\
\hline HAVEN NO. 2 & wI & 900 & $w$ & $6 / 89$ \\
\hline JAMESPORT I & NYY & 1150 & $w$ & $7 / 89$ \\
\hline JAMESPORT 2 & MIY & 1150 & $w$ & $7 / 90$ \\
\hline KOSHKONONG 1 & 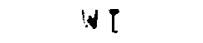 & 900 & $w$ & $2 / 85$ \\
\hline KOSHKONONG 2 & $\mathbb{N}$ & 900 & $w$ & $7 / 86$ \\
\hline NEP 1 & HI & 1150 & $w$ & $10 / 84$ \\
\hline NEP 2 & RI & 1150 & $w$ & $1 n / 86$ \\
\hline NEW HAVEN 2 & NYY & 1250 & $C E$ & $12 / 94$ \\
\hline SEARS ISLE & ME & 1150 & $\omega$ & $0 / 91$ \\
\hline SO DADE 1 & $F !$ & 1100 & H & $0 / 91$ \\
\hline SO DADE? & $F L$ & 1100 & $w$ & $0 / 91$ \\
\hline STERL ING & NY & 1150 & $w$ & $4 / 91$ \\
\hline SUNDESERT ! & CA & 974 & $w$ & $4 / 84$ \\
\hline SUNDESERT 2 & $C, \Delta$ & 974 & $\Downarrow$ & $1 / 86$ \\
\hline SURRY 3 & VA & 959 & RW & $4 / 86$ \\
\hline SURRY 4 & $V \Delta$ & 859 & $8 w$ & $4 / 87$ \\
\hline TYRONE 1 & 6 I & 1150 & $\omega$ & $4 / 84$ \\
\hline
\end{tabular}


TABLE A-4. U.S. LWR Commercial Reactors - PWR

\begin{tabular}{|c|c|c|c|c|}
\hline & \multicolumn{2}{|c|}{ INDEF INITE } & & $\begin{array}{c}\text { PLANNED } \\
\text { ATE }\end{array}$ \\
\hline PLANT NAME & $\begin{array}{l}\text { LOCATION } \\
\text { STATE }\end{array}$ & $\begin{array}{c}\text { CAPACITY } \\
\text { MWE }\end{array}$ & MANUF ACTIIRER & $\begin{array}{l}\text { ONI- } \\
\text { LINE }\end{array}$ \\
\hline 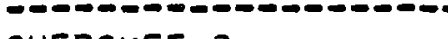 & & & & \\
\hline CHEROKEE 3 & sc & 1290 & $C E$ & $1 / 91$ \\
\hline NORTH ANNA 4 & VA & 907 & $8 w$ & $4 / 87$ \\
\hline NORTH ANNA 3 & VA & 907 & RW & $4 / 96$ \\
\hline FORKED RIVER 1 & $\mathrm{NJ}$ & $\begin{array}{l}1070 \\
1295\end{array}$ & CE & $1 ? / 83$ \\
\hline
\end{tabular}


TABLE A-5. U.S. LWR Commercial Reactors - BWR

$$
\text { ON-LINE/NOWN }
$$

\begin{tabular}{|c|c|c|c|c|c|}
\hline PLANT NAME & $\begin{array}{c}\text { LOCATION } \\
\text { STATF }\end{array}$ & $\begin{array}{c}\text { CAPACITY } \\
\text { HWF }\end{array}$ & MANUFACTIJRER & $\begin{array}{l}\text { DATE } \\
\text { ON- } \\
\text { LINE }\end{array}$ & $\begin{array}{l}\text { DATE } \\
\text { SHUT } \\
\text { DOWN }\end{array}$ \\
\hline$---\infty-\infty-\infty-\infty-\infty-\infty-\infty$ & $--\infty-\infty-\infty$ & $-0---0-0$. & 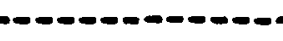 & - & $-0-0$ \\
\hline BIG ROCK POINT & MI & $7 ?$ & GE & $3 / 63$ & \\
\hline RONUS & $P R$ & 16 & CE & $6 / 65$ & $06 / 68$ \\
\hline AROWNS FERRY 1 & $\Delta L$ & 1065 & GE & $9 / 74$ & \\
\hline BROWNS FERRY 2 & $\Delta L$ & 1065 & GE & $3 / 75$ & \\
\hline BROWNS FERRY 3 & $\Delta L$ & 1065 & GE & $3 / 77$ & \\
\hline BRUNSWICK 1 & NC & 821 & GE & $3 / 77$ & \\
\hline BRUNSWICK 2 & NC & 821 & GE & $11 / 75$ & \\
\hline COOPER & $\mathrm{NH}$ & 778 & GE & $7 / 74$ & \\
\hline DRESDEN 1 & IL & 200 & GE & $7 / 60$ & \\
\hline DRESDEN ? & IL & 794 & GE & $8 / 70$ & \\
\hline DRESOEN 3 & IL & 794 & GE & $11 / 71$ & \\
\hline $\begin{array}{l}\text { DUANE ARNOLD } \\
\text { FITZPATRICK }\end{array}$ & $\begin{array}{l}\text { IA } \\
\text { NY }\end{array}$ & $\begin{array}{l}538 \\
821\end{array}$ & $\begin{array}{l}G E \\
G E\end{array}$ & $\begin{array}{l}2 / 75 \\
7 / 75\end{array}$ & \\
\hline EOWIN I HATCH I & GA & 786 & $G \bar{E}$ & $12 / 75$ & \\
\hline EDWIN I HATCH 2 & GA & 784 & $G E$ & $0 / 79$ & \\
\hline HUMBOLDT RAY 3 & $C \Delta$ & 65 & GE & $9 / 63$ & $7 / 76$ \\
\hline LACROSSE & WI & $5 n$ & $\Delta L$ & $9 / 69$ & \\
\hline MILLSTONF 1 & $C T$ & 560 & GE & $3 / 71$ & 106 \\
\hline MONT ICELL? & $M N$ & 545 & $G E$ & G/71 & \\
\hline NINE MILE PT 1 & A. $Y$ & 520 & GE & $12 / 69$ & \\
\hline OYSTER CREEK 1 & N.J & $65 n$ & GE & $12 / 69$ & \\
\hline PATHF INDER & SD & 58 & $\triangle C$ & $6 / 64$ & $10 / 67$ \\
\hline PEACH BOTTOM 2 & $P \Delta$ & 1065 & GE & $7 / 74$ & \\
\hline DEACH BOTTOM 3 & PA & 1065 & GE & $1 ? / 74$ & \\
\hline PILGRIM I & MA & 655 & GE & $12 / 72$ & \\
\hline DUAD CITIES 1 & II & 789 & GE & $9 / 72$ & \\
\hline DUAD CITIES ? & IL & 789 & GE & $10 / 72$ & \\
\hline VERMONT YANKEE & $V T$ & 514 & GE & $11 / 72$ & \\
\hline
\end{tabular}


TABLE A-6. U.S. LWR Commercial Reactors - BWR

PROJECTED

\begin{tabular}{|c|c|c|c|c|}
\hline PLANT NAME & $\begin{array}{l}\text { LOCATION } \\
\text { STATE }\end{array}$ & $\begin{array}{l}\text { CAPACITY } \\
\text { MWE }\end{array}$ & MANUF ACTURER & $\begin{array}{c}\text { D. ANNED } \\
\text { OATE } \\
\text { ONE } \\
\text { LINE }\end{array}$ \\
\hline$m-\infty-\infty-\infty-\infty-\infty-\infty-\infty$, & $n-\infty-\infty$ & & $0-0-0-0-0-0$ & \\
\hline BAILLY N 1 & IN & 644 & GE & $5 / 87$ \\
\hline BLACK FOX 1 & OK & 1150 & GE & $7 / 85$ \\
\hline BLACK FOX 2 & OK & 1150 & GE & $7 / 88$ \\
\hline CLINTON 1 & IL & 933 & GE & $3 / 83 *$ \\
\hline CLINTON 2 & IL & 933 & $G E$ & $6 / 88$ \\
\hline FERMI 2 & $M I$ & 1093 & GE & $3 / 82$ \\
\hline GRAND GULF 1 & MS & 1250 & GE & $4 / 83 *$ \\
\hline GRAND GULF 2 & MS & 1250 & GE & $4 / 86$ * \\
\hline HOPE CREEK 1 & NJ & 1067 & GE & $5 / 86 *$ \\
\hline HOPE CREEK 2 & NJ & 1067 & GE & $5 / 89 *$ \\
\hline LASALLE 1 & IL & 1078 & GE & $5 / 81$ \\
\hline LASALLE 2 & IL & 1078 & GE & $5 / 82^{*}$ \\
\hline LIMERICK I & $P A$ & 1055 & GE & $4 / 83 *$ \\
\hline LIMERICK 2 & $P A$ & 1055 & GE & $4 / 85$ \\
\hline NINE MILE POINT $Z$ & NY & 1099 & GE & $10 / 86$ \\
\hline PERRY I & $O H$ & 1205 & GE & $5 / 84$ \\
\hline PERRY Z & $\mathrm{OH}$ & 1205 & GE & $5 / 88$ \\
\hline PHIPPS BEND 1 & $T N^{4}$ & 1233 & GE & $3 / 87$ \\
\hline RIVER BEND 1 & La & 934 & GE & $4 / 84$ \\
\hline RIVER GEND 2 & L1 & 934 & GE & $0 / 90$ \\
\hline SHOREHAM & NY & A19 & GE & $3 / 83 *$ \\
\hline SKAGIT 1 & $w \Delta$ & 1277 & GF. & $1 / 90$ \\
\hline SKAGIT? & Wa & 1277 & $G E$ & $1 / 92$ \\
\hline SUSRUEHANAIA 1 & $P A$ & 1050 & GE & $7 / 82$ * \\
\hline SUSQUEHANNA ? & $P \Delta$ & 1050 & GE & $7 / 83 *$ \\
\hline WPPSS-2 & WA & 1093 & GE & $9 / 81$ \\
\hline ZIMMER I & $\mathrm{OH}$ & 810 & GE. & $4 / 82$ * \\
\hline
\end{tabular}

* Date is later than reported in PNL-3317-1. 
TABLE A-7. U.S. LWR Commercial Reactors - BWR CANCELLED

\begin{tabular}{|c|c|c|c|c|}
\hline PLANT NAME & $\begin{array}{c}\text { LOCATION } \\
\text { STATE }\end{array}$ & $\begin{array}{l}\text { CAPACITY } \\
\text { MWE }\end{array}$ & MANUF ACTUREP & $\begin{array}{l}\text { L ANNED } \\
\text { DATE } \\
\text { ONE } \\
\text { LINE }\end{array}$ \\
\hline 8ARTON 1 & $\Delta L$ & 1159 & GF & $0 / 90$ \\
\hline BARTOA Z & $\Delta L$ & 1159 & GE & $0 / 90$ \\
\hline DOUGLAS PT 1 & m & 1146 & GE & $3 / 87$ \\
\hline DOUGLAS DT $Z$ & in & 1146 & GE & $3 / 91$ \\
\hline MONTAGUE 1 & MA & 1150 & GE & $4 / 89$ \\
\hline MONTAGUE? & $M A$ & 1150 & $G \varepsilon$ & $1 / 90$ \\
\hline PACIFIC GAS/ELEC 1 & $c a$ & 1200 & GE & $n / 91$ \\
\hline PACIFIC GAS/ELEC 2 & $C \Delta$ & 1200 & GE & $0 / 91$ \\
\hline 7IMMER 2 & $n H$ & 1170 & GE & $n / 87$ \\
\hline
\end{tabular}


TABLE A-8. U.S. LWR Commercial Reactors - BWR

\begin{tabular}{|c|c|c|c|c|}
\hline & \multicolumn{2}{|c|}{ INDEFINITE } & & PLANNED \\
\hline PLANT NAME & $\begin{array}{l}\text { LOCATION } \\
\text { STATE }\end{array}$ & $\begin{array}{l}\text { CAPACITY } \\
\text { MWE }\end{array}$ & MANUF ACTURER & $\begin{array}{l}\text { ONE } \\
\text { LINE }\end{array}$ \\
\hline $\begin{array}{l}\text { HARTSVILLE } \\
\text { HARTSVILLE } \\
\text { HART } \\
\text { HARTSVILLE } \\
\text { BI } \\
\text { HARTSVILLE } \\
\text { PHIPPS BEND }\end{array}$ & $\begin{array}{l}\text { TN } \\
\text { TN } \\
\text { TN } \\
\text { TM } \\
\text { TiN }\end{array}$ & $\begin{array}{l}1233 \\
1233 \\
1233 \\
1233 \\
1233\end{array}$ & $\begin{array}{l}G F \\
G E \\
G E \\
G E \\
G E\end{array}$ & $\begin{array}{l}7 / 86 \\
7 / 87 \\
6 / 89 \\
6 / 90 \\
8 / 89\end{array}$ \\
\hline
\end{tabular}


TABLE A-9. U.S. LWR Commercial Reactors

Announced as of 1-1-77

since Cancelled

\begin{tabular}{|c|c|c|c|}
\hline Project/Location & Owner & $\begin{array}{l}\text { Capacity } \\
\text { Net } \\
\text { (MWe) }\end{array}$ & $\begin{array}{l}\text { Public } \\
\text { Annc'd }\end{array}$ \\
\hline $\begin{array}{l}\text { Fulton Generating Station } \\
\text { Unit } 1 \text { (PA) }\end{array}$ & Philadelphia Electric Co. & 1160 & $8 / 71$ \\
\hline $\begin{array}{l}\text { Fulton Generating Station } \\
\text { Unit } 2 \text { (PA) }\end{array}$ & Philadelphia Electric Co. & 1160 & $8 / 71$ \\
\hline $\begin{array}{l}\text { Summit Power Station } \\
\text { Unit } 1 \text { (DE) }\end{array}$ & Delmarva Power \& Light Co. & 1200 & $12 / 76$ \\
\hline $\begin{array}{l}\text { San Joaquin Nuclear } \\
\text { Project } 1 \text { (CA) }\end{array}$ & $\begin{array}{l}\text { LA Dept. of Water, PG\&E } \\
\text { SCE, SDE \&G, COWR }\end{array}$ & 1300 & $3 / 74$ \\
\hline $\begin{array}{l}\text { San Joaqu in Nuclear } \\
\text { Project } 2 \text { (CA) }\end{array}$ & $\begin{array}{l}\text { LA Dept. of Water, PG\&E } \\
\text { SCE, SOE \&G, CDWR }\end{array}$ & 1300 & $3 / 74$ \\
\hline $\begin{array}{l}\text { San Joaquin Nuclear } \\
\text { Project } 3 \text { (CA) }\end{array}$ & $\begin{array}{l}\text { LA Dept. of Water, PG\&E } \\
\text { SCE, SDE } \& G \text {, CDWR }\end{array}$ & 1300 & $3 / 74$ \\
\hline $\begin{array}{l}\text { San Joaquin Nuclear } \\
\text { Project } 4 \text { (CA) }\end{array}$ & $\begin{array}{l}\text { LA Dept. of Water, PG\&E } \\
\text { SCE, SOE\&G, CDWR }\end{array}$ & 1300 & $3 / 74$ \\
\hline $\begin{array}{l}\text { Rancho Seco Nuclear } \\
\text { Gen. Stat. No. } 2 \text { (CA) }\end{array}$ & Sacramento Municipal & 1100 & $9 / 74$ \\
\hline $\begin{array}{l}\text { Nebraska Public Power } \\
\text { (NB) }\end{array}$ & Nebraska Public Power & 1100 & $1 / 75$ \\
\hline $\begin{array}{l}\text { Mid-Hudson East } 1 \\
\text { (NY) }\end{array}$ & $\begin{array}{l}\text { Empire State Power } \\
\text { Resources }\end{array}$ & 1300 & $4 / 75$ \\
\hline $\begin{array}{l}\text { Mid-Hudson East } 2 \\
\text { (NY) }\end{array}$ & $\begin{array}{l}\text { Empire State Power } \\
\text { Resources }\end{array}$ & 1300 & $4 / 75$ \\
\hline $\begin{array}{l}\text { Mid-Hudson West } 1 \\
\text { (NY) }\end{array}$ & $\begin{array}{l}\text { Empire State Power } \\
\text { Resources }\end{array}$ & 1300 & $4 / 75$ \\
\hline $\begin{array}{l}\text { Shoreham West } 1 \\
\text { (NY) }\end{array}$ & $\begin{array}{l}\text { Empire State Power } \\
\text { Resources }\end{array}$ & 1300 & $4 / 75$ \\
\hline $\begin{array}{l}\text { Shoreham West } 2 \\
\text { (NY) }\end{array}$ & $\begin{array}{l}\text { Empire State Power } \\
\text { Resources }\end{array}$ & 1300 & $4 / 75$ \\
\hline $\begin{array}{l}\text { St. Lawrence } 1 \\
(N Y)\end{array}$ & $\begin{array}{l}\text { Empire State Power } \\
\text { Resources }\end{array}$ & 1300 & $4 / 75$ \\
\hline $\begin{array}{l}\text { St. Lawrence } 2 \\
(N Y)\end{array}$ & $\begin{array}{l}\text { Empire State Power } \\
\text { Resources }\end{array}$ & 1300 & $4 / 75$ \\
\hline $\begin{array}{l}\text { Eastern Desert } 1 \\
\text { (CA) }\end{array}$ & $\begin{array}{l}\text { Southern California } \\
\text { Edison Co. }\end{array}$ & 1000 & $7 / 76$ \\
\hline $\begin{array}{l}\text { Eastern Desert } 2 \\
\text { (CA) }\end{array}$ & $\begin{array}{l}\text { Southern Californid } \\
\text { Edison Co. }\end{array}$ & 1000 & $7 / 76$ \\
\hline
\end{tabular}




\section{DISTRIBUTION}

No. of

Copies

OFFSITE

UNITED STATES

A. A. Churm

DOE Chicago Patent Group

9800 South Cass Avenue

Argonne, IL 60439

10 Department of Energy

NEW, B-107, HQ

Washington, DC 20545

Attn: S. T. Brewer

C. A. Heath

M. J. Lawrence

E. F. Mastal

G. Oerte 1

R. W. Ramsey, Jr.

R. Romatowski

J. W. Rowen

V. G. Trice

E. Wahlquist

William Barnard

Office of Technology Assessment

U.S. Congress

Washington, DC 20510

2 DOE Savannah River Operations Office

P.0. Box A

Aiken, SC 29801

Attn: M. C. Kirkland

R. P. Whitfield

3 DOE Technical Information Center

2 EG\&G Idaho

P.0. Box 1625

Idaho, ID 83415

Attn: C. A. Aquilana

G. B. Levin
No. of

Copies

4 DOE Albuquerque Operations Office

P.0. Box 5400

Albuquerque, NM 87185

Attn: J. L. Bellows

R. Campbe 11

M. L. Matthews

D. T. Schueller

Office of Nuclear Waste Isolation

Battelle Memorial Institute

Attn: Beverly Rawles

$505 \mathrm{King}$ Avenue

Columbus, $\mathrm{OH} 43201$

R. W. Peterson

Technical Support

Office of Nuclear Waste Isolation

Battelle Memorial Institute

505 King Avenue

Columbus, $\mathrm{OH} 43201$

C. M. Brown

Rockwe 11

Rocky Flats Area Office

P.0. Box 928

Golden, CO 80401

Lawrence J. Smith

TRU Waste Systems Office

Bldg. T-790

Rockwe 11 International

Rocky Flats Plant

P.0. Box 464

Golden, CO 80401 
No. of

Copies

5 E.I. Du Pont de Nemours Co. Savannah River Laboratory Aiken, SC 29801

Attn: W. H. Baker

W. R. Cornman

J. L. Cranda 11

F. King

P. H. Permar

E. L. Keller

DOE Oak Ridge Operations Office P.0. Box E

Oak Ridge, TN 37830

2 Oak Ridge National Laboratory

P.0. Box $X$

Oak Ridge, TN 37830

Attn: J. 0. Blomeke

K. J. Notz

3 Sandia Laboratories

P.0. Box 5800

Albuquerque, NM 87185

Attn: G. C. Allen, Jr.

R. Jefferson

E. Minor

J. W. Bartlett

The Analytical Sciences Corp.

6 Jacob Way

Reading, MA 01867

R. L. Murray

Department of Nuclear

Engineering

North Carolina State University

Raleigh, NC 27650

F. K. Pittman

3508 Sagecrest Terrace

Ft. Worth, TX 76109

R. E. Burns

2412 Cedar Park Drive

Port Angeles, WA 98362
No. of

Copies

M. T. Johnson

Advanced Energy Systems Division

Power Systems Company

Westinghouse Electric Corp.

Box 10864

Pittsburgh, PA 25236

A. J. O'Donne11, Bechte 1 Nationa 1, Inc. P.0. Box 3965

San Francisco, CA 94119

ONSITE

5 DOE Richland Operations Office

P. A. Craig

R. B. Goranson

H. E. Ransom

J. J. Schreiber

M. W. Shupe

3 Rockwe 11 Hanford Operations

R. A. Deju

C. G. Evans

D. D. Wodrich

3 United Nuclear Corporation

C. G. Jones

R. L. Miller

J. F. Nemec

32 Pacific Northwest Laboratory
W. B. Andrews
J. C. Bower
J. B. Brown
R. A. Burnett 
No. of

Copies

T. D. Chikalla

R. L. Enge 1

R. M. Fleischman

J. H. Jarrett

A. B. Johnson

M. R. Kreiter

L. T. Lakey

R. C. Liikala

R. P. Marsha 11

J. E. Mende 1

E. T. Merrill

R. E. Nightingale/J. L. McElroy
No. of

Copies
C. R. Palmer
A. M. Platt
J. A. Powe 11
R. E. Rhoads
J. V. Robinson (3)
C. W. Roll and
A. M. Sutey
C. M. Unruh
R. D. Widrig
L. D. Williams
Technical Information (3)
Publishing Coordination CO (1) 
. 